\title{
Stochastic multiscale analysis in hydrodynamic lubrication
}

\author{
A. Waseem ${ }^{1}$, J. Guilleminot ${ }^{2}, * \dagger$ (i) and İ. Temizer ${ }^{1}$ \\ ${ }^{1}$ Department of Mechanical Engineering, Bilkent University, Ankara, 06800, Turkey \\ ${ }^{2}$ Université Paris-Est, Laboratoire Modélisation et Simulation Multi Echelle, Marne la Vallée, 77454, France
}

\begin{abstract}
SUMMARY
A stochastic multiscale analysis framework is developed for hydrodynamic lubrication problems with random surface roughness. The approach is based on a multi-resolution computational strategy wherein the deterministic solution of the multiscale problem for each random surface realization is achieved through a coarse-scale analysis with a local upscaling that is achieved through homogenization theory. The stochastic nature of this solution because of the underlying randomness is then characterized through local and global quantities of interest, accompanied by a detailed discussion regarding suitable choices of the numerical parameters in order to achieve a desired stochastic predictive capability while ensuring numerical efficiency. Finally, models of the stochastic interface response are constructed, and their performance is demonstrated for representative problem settings. Overall, the developed approach offers a computational framework, which can essentially predict the significant influence of interface heterogeneity in the absence of a strict scale separation. Copyright (C) 2017 John Wiley \& Sons, Ltd.
\end{abstract}

Received 9 December 2016; Revised 8 February 2017; Accepted 1 March 2017

KEY WORDS: stochastic analysis; multiscale simulation; hydrodynamic lubrication; Reynolds equation; homogenization

\section{INTRODUCTION}

Lubrication is one of the major branches of tribology that is relevant to a broad range of interface problems, from industrial applications in the design of moving machinery and tire tread patterns to biological scenarios that underlie the healthy functioning of the synovial joints and the digestive system $[1,2]$. In general, the physics at the interface is highly complex and often necessitates the consideration of a mixed regime where multiple contact regions may exist across the interface. Presently, with a view towards the multiscale mechanics of the interface, attention will be confined to the hydrodynamic regime where the interacting surfaces are fully separated by a fluid film, an assumption that has classically served as a starting point for the consideration of the mixed regime as well. In either regime, with particular relevance to industrial applications, the inevitable presence of surface roughness leads to a heterogeneous film distribution at the interface. It is now well known that if the surfaces are in sufficient proximity, then roughness can induce quantitatively and qualitatively significant changes in the macroscopic lubrication response of the interface in comparison with the response from a homogeneous film distribution, that is, one where the film thickness varies only across macroscopic dimensions. The aim of this work is to introduce a stochastic multiscale analysis methodology for this hydrodynamic lubrication problem with random roughness. This methodology will be based upon established two-scale formulations that characterize the influence of surface roughness on the macroscopic response [3,4], which will be applied in the spirit of multi-resolution approaches proposed in multiscale mechanics [5, 6]. In order to highlight the need

\footnotetext{
*Correspondence to: Johann Guilleminot, Université Paris-Est, Laboratoire Modélisation et Simulation Multi Echelle, 77454 Marne la Vallée, France.

†E-mail: johann.guilleminot@u-pem.fr
} 
for such a novel computational methodology, some of the key contributions towards the development of relevant two-scale formulations will first be briefly reviewed in the following, specifically those which address the most general setting where both of the interacting surfaces could be rough (bilateral roughness) and both could be moving (bilateral motion).

\subsection{Overview of deterministic two-scale approaches}

Two of the earliest key contributions towards addressing the influence of surface roughness are [7] and [8] where roughness effects were incorporated through flow factors that contain statistical information about the two surfaces. The form of the macroscopic governing equation that was proposed in these works was specific to surfaces that macroscopically display an isotropic or a very restricted class of anisotropic responses. This was pointed out in $[9,10]$ where the macroscopic formulation that is appropriate to an unrestricted anisotropic response was established, essentially by replacing the scalar flow factors by constitutive tensorial coefficients. A detailed derivation of this unrestricted anisotropic formulation through an averaging-based approach was more recently provided by [4]. The overall formulation proposed therein is equivalent to the formulation that is delivered by a rigorous homogenization framework based on asymptotic expansion, which was outlined in [3] for the general case of bilateral roughness and motion. In particular, both formulations properly account for rapid variations in the local film thickness with respect to time, and the closure problems constructed in [4] can readily be expressed in the form of the cell problems of homogenization as derived in [3], thereby leading to identical forms of the macroscopic equation, which governs the interface response [11]. Consequently, both formulations are equally effective in accounting for interface heterogeneity and describing the link between the macroscopic and the microscopic scales, that is, they provide a complete two-scale formulation of the problem.

\subsection{Scope of the present work}

A number of remarks regarding the scope of the present work are relevant at this point. First, the homogenization-based approach, which constitutes the starting point in the present study, is based on the framework of [3]. Although the derivation of this framework explicitly assumes a space-time periodic film thickness, such an assumption is not invoked in the work of [4] that is representative of earlier averaging-based approaches, which explicitly address non-periodic roughness. However, two-scale formulations relying on asymptotic expansions (as in the periodic setting) can still be applied in averaging-based micromechanics of random media [12], provided that the assumptions of stationarity and ergodicity hold [13]. For this reason, homogenization results are routinely applied to non-periodic roughness as well $[11,14,15]$ without the need for a rederivation of the two-scale formulation. Nevertheless, it is highly intriguing to ask whether a non-periodic setting leads to shortcomings in the predictive capabilities of these two-scale formulations. The identification and addressing of potential shortcomings is a central goal of the present study in the context of multiscale hydrodynamic lubrication problems.

Second, the present work will be carried out, following most earlier works, via a strong formulation of the problem. The fact that equivalent weak formulations may be stated in the context of homogenization was already pointed out in [16], and possible mathematical advantages of such an approach have been discussed in [17]. These advantages are particularly relevant to nonlinear problems [18]. Presently, the implementation of the proposed two-scale framework will be realized via the finite element method. Consequently, although not pursued, a possibility for restating this framework in a weak form naturally exists in a numerical setting [19].

Third, the general setting of bilateral interface roughness and motion leads to oscillations in the macroscopic solution when commensurate periodic surfaces are involved [3]. These oscillations have a non-negligible amplitude, and they occur at a very high frequency, which renders the solution of the macroscopic problem prohibitively expensive. To alleviate this expense, a time-averaging approach may be invoked [4]. However, the oscillations disappear as soon as the rough surfaces are incommensurate, with or without periodicity [11]. Randomly rough surfaces are always incommensurate. As such, both the microscopic and the macroscopic physics are qualitatively similar to the case of unilateral surface roughness. Moreover, in this setting, bilateral surface motion can easily be accounted 
for, based on the setup with unilateral surface motion [20]. Consequently, only unilateral roughness (lower surface) and motion (upper surface) will be considered in this work, which will eliminate the appearance of time on the microscale. Time-dependence on the macroscale may remain as an indication of a possibility for mean film thickness variation. Because such a variation essentially acts as a deterministic time-dependent source term in the macroscopic differential equation, it does not influence the construction of the stochastic and the deterministic frameworks. Consequently, it will also be omitted. It should be remarked that the bilateral case is quantitatively not identical to the unilateral case. However, the stochastic two-scale approach to be developed will be indicative of the approach that can be taken as the starting point for addressing the bilateral case as well.

Finally, following the works cited earlier, which address the two-scale problem of hydrodynamic lubrication, the physics of the microscopic problem is modeled with the Reynolds equation, and therefore, the macroscopic problem also admits a Reynolds-type equation. The assumptions that govern the derivation of the Reynolds equation are well known $[1,2]$. On the other hand, in the context of homogenization, a crucial assumption is that the ratio of a representative roughness wavelength to a representative macroscopic dimension is vanishingly small. It has been shown in [21] that if the ratio of the roughness wavelength to the mean film thickness is too small, then a Reynolds-type macroscopic equation is still implied, but instead of the two-dimensional Reynolds equation, it may be more appropriate to model the physics of the microscopic problem with the three-dimensional Stokes equation or even assume a simple scenario where the film thickness is assigned its locally minimum value throughout the whole interface. Consequently, it is presently assumed that the wavelength is small enough to enable a two-scale analysis of the problem but large enough for the Reynolds equation to be valid on the microscale, which is often referred to as Reynolds roughness.

\section{DETERMINISTIC HOMOGENIZATION WITH SCALE SEPARATION}

\subsection{Periodic setting}

Let $\varepsilon$ indicate the ratio of the roughness wavelength to a macroscopic dimension. The absolute position vector across the macroscopic interface geometry will be denoted by $\boldsymbol{x}_{\varepsilon}$. The interface is assigned a heterogeneous lubrication film, with thickness $h_{\varepsilon}\left(\boldsymbol{x}_{\varepsilon}\right)$. For the classical derivation of the two-scale deterministic homogenization framework [3], one invokes scale separation such that $\varepsilon \ll 1$. It is then advantageous to introduce independent macroscopic and microscopic position vectors $\boldsymbol{x}$ and $\boldsymbol{y}$, respectively, using which one expresses the absolute position vector as

$$
\boldsymbol{x}_{\varepsilon}=\boldsymbol{x}+\varepsilon \boldsymbol{y} .
$$

The macroscopic film thickness $h_{0}$ is a local area average of the absolute film thickness $h_{\varepsilon}$, and it varies only with $\boldsymbol{x}$. In a unilateral setting, $h_{\varepsilon}>0$ may be assigned the relatively general expression

$$
h_{\varepsilon}\left(\boldsymbol{x}_{\varepsilon}\right)=h(\boldsymbol{x}, \boldsymbol{y})=h_{0}(\boldsymbol{x})-\bar{h}(\boldsymbol{y}),
$$

where $\bar{h}$ indicates thickness variation (with zero mean) because of the microscopic roughness of the stationary lower surface. The heterogeneous fine-scale problem is then stated as

$$
-\nabla_{\boldsymbol{x}_{\varepsilon}} \cdot \boldsymbol{q}_{\varepsilon}\left(\boldsymbol{x}_{\varepsilon}\right)=0
$$

where, indicating the constant fluid viscosity by $\mu$, the absolute flux $\boldsymbol{q}_{\varepsilon}\left(\boldsymbol{x}_{\varepsilon}\right)$ depends on the gradient $\boldsymbol{g}_{\varepsilon}=\nabla_{\boldsymbol{x}_{\varepsilon}} p_{\varepsilon}$ of the absolute interface pressure $p_{\varepsilon}$ as well as on the tangential velocity $\overline{\boldsymbol{U}}$ of the smooth upper surface:

$$
\boldsymbol{q}_{\varepsilon}=-\frac{h_{\varepsilon}^{3}}{12 \mu} \boldsymbol{g}_{\varepsilon}+\frac{h_{\varepsilon}}{2} \overline{\boldsymbol{U}}
$$

The two-scale formulation of the problem now follows by substituting an asymptotic expansion of the pressure 


$$
p_{\varepsilon}\left(\boldsymbol{x}_{\varepsilon}\right)=p_{0}(\boldsymbol{x}, \boldsymbol{y})+\varepsilon p_{1}(\boldsymbol{x}, \boldsymbol{y})+\varepsilon^{2} p_{2}(\boldsymbol{x}, \boldsymbol{y})+\mathcal{O}\left(\varepsilon^{3}\right)
$$

into (2.3) and collecting equal powers of $\varepsilon$. Terms of order $\varepsilon^{-2}$ eliminate the $\boldsymbol{y}$-dependence of $p_{0}$. Indicating its gradient by $\boldsymbol{G}=\nabla_{x} p_{0}$ and upon introducing the convenient expansion

$$
p_{1}=\boldsymbol{G} \cdot \boldsymbol{\omega}-\overline{\boldsymbol{U}} \cdot \Omega
$$

in view of the linearity of the problem, terms of order $\varepsilon^{-1}$ deliver microscopic problems from which $\boldsymbol{\omega}(\boldsymbol{x}, \boldsymbol{y})=\omega_{i} \boldsymbol{e}_{i}$ and $\boldsymbol{\Omega}(\boldsymbol{x}, \boldsymbol{y})=\Omega_{i} \boldsymbol{e}_{i}$ may be solved:

$$
\frac{\partial}{\partial y_{i}}\left(\frac{h^{3}}{12 \mu} \delta_{i j}+\frac{h^{3}}{12 \mu} \frac{\partial \omega_{j}}{\partial y_{i}}\right)=0 \quad, \quad \frac{\partial}{\partial y_{i}}\left(\frac{h}{2} \delta_{i j}+\frac{h^{3}}{12 \mu} \frac{\partial \Omega_{j}}{\partial y_{i}}\right)=0 .
$$

Here, $\boldsymbol{e}_{i}(i=1,2)$ indicates the orthonormal basis vectors and $\delta_{i j}$ is the Kronecker delta. The crucial assumption of periodicity is enforced by solving these problems on a periodic cell $\mathcal{Y}$ subject to periodic boundary conditions on $\partial \mathcal{Y}$ and, thereby, assuming that all terms, which have an explicit dependence on $\boldsymbol{y}$, are $\mathcal{Y}$-periodic. Indicating the average over $\mathcal{Y}$ with $\langle\cdot\rangle$, one may finally define macroscopic tensorial coefficients $\boldsymbol{A}(\boldsymbol{x})$ and $\boldsymbol{C}(\boldsymbol{x})$ with components

$$
A_{i j}=\left\langle\frac{h^{3}}{12 \mu} \delta_{i j}+\frac{h^{3}}{12 \mu} \frac{\partial \omega_{j}}{\partial y_{i}}\right\rangle \quad, \quad C_{i j}=\left\langle\frac{h}{2} \delta_{i j}+\frac{h^{3}}{12 \mu} \frac{\partial \Omega_{j}}{\partial y_{i}}\right\rangle .
$$

These coefficients appear in the macroscopic problem posed on a macroscopic domain, obtained by averaging terms of order $\varepsilon^{0}$, the solution of which is the homogenized interface pressure $p_{0}$ :

$$
\boldsymbol{Q}=-\boldsymbol{A} \boldsymbol{G}+\boldsymbol{C} \overline{\boldsymbol{U}} \rightarrow-\nabla_{\boldsymbol{x}} \cdot \boldsymbol{Q}=0 .
$$

\subsection{A classical transition to randomness}

In the two-scale finite element setting that is implied by homogenization, the microscopic problems are solved at each quadrature point of the macroscopic finite element mesh, in order to obtain the tensorial coefficients $\boldsymbol{A}$ and $\boldsymbol{C}$ for the integration of the macroscopic weak form that subsequently delivers $p_{0}$. From a visualization point of view, one may imagine that a single, well-defined surface microstructure is assigned to each macroscopic quadrature point. This microstructure is represented by the periodic $\bar{h}$ variation across $\mathcal{Y}$. It is sufficient to pick a single period, so that $\mathcal{Y}$ corresponds to a unit-cell in this case.

A straightforward relaxation of this framework to a non-periodic setting is typically carried out by assigning $\mathcal{Y}$ a single realization of random $\bar{h}$. Note that this single realization may or may not satisfy periodicity on $\partial \mathcal{Y}$ - a non-periodic realization is essentially equivalent to a periodic realization with jumps in $\bar{h}$ within $\mathcal{Y}$, and in both cases, the macroscopic tensorial coefficients can still be defined through (2.8). It is then argued that $\mathcal{Y}$ must be sufficiently large in order to be statistically representative of the surface characteristics, that is, the ratio of the wavelength to the cell size must be vanishingly small. A sufficiently large $\mathcal{Y}$ minimizes the scatter in the values of the tensorial coefficients within an ensemble of realizations, thereby leading to a unique macroscopic solution $p_{0}$ even for a random surface microstructure realization $[11,15]$. In other words, the macroscopic problem still admits a deterministic representation.

On the other hand, for the described periodic-to-random transition argument to be valid, a key condition must simultaneously be met: the statistically representative cell size $(\ell)$ must be sufficiently small with respect to a representative macroscopic dimension $(L)$. Such a dimension is naturally provided by a distance over which the macroscopic solution changes appreciably. When the ratio $\ell / L$ is small across the whole domain, the solution $p_{0}$ to the two-scale problem will accurately match the solution $p_{\varepsilon}$ to the original heterogeneous problem for any realization of roughness across the entire interface. If this condition is not met, $p_{\varepsilon}$ may be significantly dominated by local features in the heterogeneous film thickness $h_{\varepsilon}$ such that appreciably large differences between $p_{0}$ and $p_{\varepsilon}$ may be observed and, hence, a deterministic two-scale formulation ceases to be meaningful. 


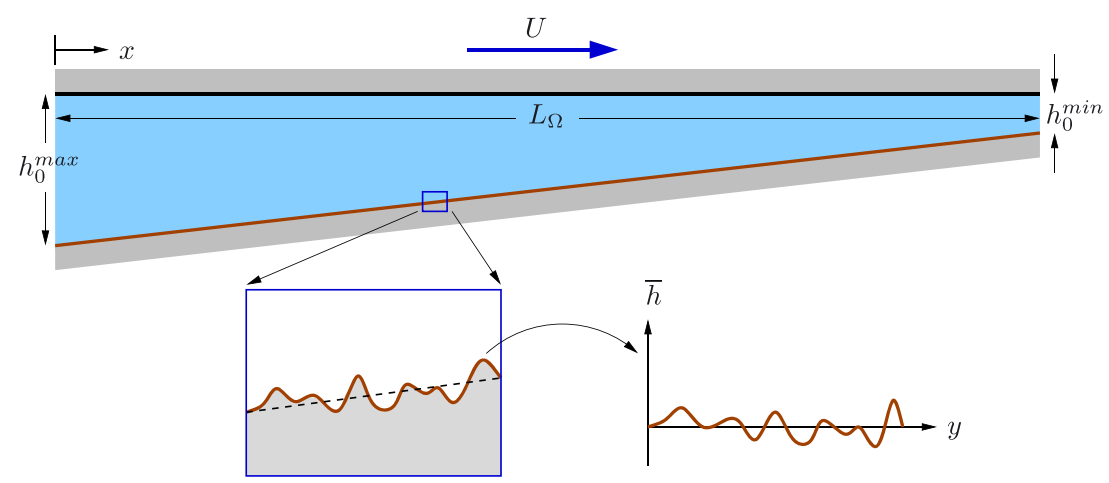

Figure 1. The classical wedge problem is depicted in one dimension (not drawn to scale). Because of the unilateral setting, the microscopically smooth top surface is moving and the rough bottom surface is stationary - see (2.2) for the film thickness decomposition. On the boundary, the pressure is set to zero.

Clearly, in addition to the described complexity, typical in-plane and out-of-plane random roughness characterization measures such as root-mean-square roughness and autocorrelation functions, respectively, can also display random variations across an interface. Such variations would also render the application of the described deterministic framework highly questionable.

In the following section, a stochastic two-scale framework is described to address such shortcomings in the deterministic approach, primarily concentrating on the former complexity. To the best of the authors' knowledge, a rigorous framework of comparable scope does not exist in the literature - see [22] for a recent relevant study that concentrates on the interface permeability (the latter being modeled as a log-normal random variable with a point-wise statistical fitting involving subscale simulations; the specific construction of an information-theoretic model for tensor-valued permeability random fields can be found in [23]).

\section{STOCHASTIC HOMOGENIZATION}

Let $\Omega:=\left[0, L_{1}\right] \times\left[0, L_{2}\right]$ be the macroscopic domain under consideration. In this work, although the methodological aspects of the stochastic framework are problem independent, the macroscopic flow problem is defined as a wedge problem, subject to zero pressure boundary conditions (Figure 1). Here, the coarse-scale position will be denoted by $x \in \Omega$ and the roughness-level fine-scale position by $\boldsymbol{y} \in \Omega$, the latter playing a role equivalent to $\boldsymbol{x}_{\varepsilon}$ because of the upscaling approach to be introduced in Section 3.2.1. Hence, the mean film thickness is described by the deterministic function $\boldsymbol{x} \mapsto h_{0}(\boldsymbol{x})$ given by

$$
h_{0}(\boldsymbol{x})=\frac{h_{0}^{\min }-h_{0}^{\max }}{L_{1}} x_{1}+h_{0}^{\max }, \quad \forall \boldsymbol{x} \in \Omega,
$$

with $h_{0}^{\min }$ and $h_{0}^{\max }$ two given real constants such that $h_{0}^{\min }<h_{0}^{\max }$. For numerical illustrations, we set $L_{1}=L_{2}=L_{\Omega}=100,\left\{h_{0}^{\min }, h_{0}^{\max }\right\}=\{0.4,1.4\}$ in $1 \mathrm{D}$ and $\{0.2,1\}$ in $2 \mathrm{D}$ (units: $[\mu \mathrm{m}]$ ). The velocity of the upper surface is $\left(\bar{U}_{1}, \bar{U}_{2}\right)=(1,0) \mathrm{m} / \mathrm{s}$, and the viscosity in (2.4) is fixed to $0.05 \mathrm{~Pa} \cdot \mathrm{s}$ in all examples. The surface roughness is further described as a random field $\{\bar{h}(\boldsymbol{y}), \boldsymbol{y} \in \Omega\}$ defined by an information-theoretic probabilistic model. The construction of the latter is specifically addressed in the next section.

\subsection{Definition of the random topography}

3.1.1. Mathematical construction. The definition of mathematical models for random surfaces has been a subject of longstanding interest in different fields of science and engineering. One 
major difficulty stems from the fact that such a definition depends on various factors (such as the underlying microstructure and the processing conditions), which make models intrinsically application-dependent (see [24] and the references therein for a survey, as well as [25] for a recent discussion). Additionally, surface characterization may involve several criteria [26, 27] that generally render the identification non-unique. From a probabilistic point of view, it should be noticed that the validation of a model should be completed on the full system of (finite-dimensional) marginal probability distributions, which is not achievable in standard experimental settings. When data are available, a classical approach to model selection then consists in choosing a labeled random field model and in subsequently discussing the plausibility of the retained assumption a posteriori - using, for example, statistical tests on either the topology or on predicted quantities of interest such as macroscopic contact properties. Common practices and assumptions have therefore fed in fundamental debates, including discussions on the fractal versus non-fractal nature of surfaces [28, 29], or on the relevance of Gaussian models [30,31]. Undoubtedly, the assumption of Gaussianity is certainly the most widely invoked, at least for the two following reasons:

- First, a Gaussian model is analytically tractable and allows useful statistics on upcrossings and excursion sets [32] to be estimated for example;

- Second, the Gaussian model is completely defined by its mean and correlation functions, hence making the identification of the hyperparameters more easily tractable.

In this contribution, and for reasons that will appear in the following, we explore the non-Gaussian case and address the construction of a probabilistic model defining the random field $\{\bar{h}(\boldsymbol{y}), \boldsymbol{y} \in \Omega\}$. The intent is to derive a prototypical model that is on the one hand consistent with known constraints and that exhibits on the other hand a low-dimensional parametrization (in the spirit of a Gaussian model) involving key multiscale features - such as variance control and spatial correlation lengths. In a global approach involving the assimilation of (experimental) data, such a prior model may subsequently be updated through a Bayesian analysis. Here, the stochastic framework is specifically derived under the constraint that the event $\left\{\bar{h}(\boldsymbol{y}) \geqslant h_{0}(\boldsymbol{x})\right\}$ is assigned a zero probability almost surely. This condition, which ensures that the two surfaces do not interpenetrate each other, will be referred to as the (physical) consistency condition in the sequel and makes a Gaussian model nonadmissible from a theoretical standpoint. While a rejection-like algorithm can be used in practice to discard realizations violating the previous property, it should further be noticed that such a strategy would result in a large rejection rate in case the stochastic field is generated over large lattices and/or exhibits moderate to large statistical fluctuations - hence increasing the overall computation time. In order to construct the stochastic model, we alternatively propose the following two-step methodology relying on information theory.

In a first stage, let $\{\xi(\boldsymbol{y}), \boldsymbol{y} \in \Omega\}$ be the centered real-valued Gaussian random field, defined by the separable periodic correlation function $\tau \mapsto \rho(\tau)$ given by

$$
\rho(\tau):=\rho_{1}\left(\tau_{1}\right) \times \rho_{2}\left(\tau_{2}\right),
$$

where $\tau$ is the lag vector and $\tau_{i} \mapsto \rho_{i}\left(\tau_{i}\right), i=1,2$ is a normalized correlation function. In this work, this function will additionally be assigned periodicity on $\partial \Omega$ in order to benefit from the same generation algorithm in a classical two-scale setting with randomness where, although not strictly required (Section 2.2), each $\bar{h}$-realization would be periodic on $\partial \mathcal{Y}$. In practice, $\tau_{i}$ may be either identified using a general Fourier expansion and periodized versions of the measurements or selected in a given class of correlation functions. For illustration purposes, $\rho_{i}$ is defined by applying a warping technique to a squared-exponential kernel [33], that is,

$$
\rho_{i}(\tau):=\exp \left\{-\frac{2}{\alpha_{i}^{2}} \sin ^{2}\left(\frac{\pi \tau}{L_{i}}\right)\right\}, \quad i=1,2 .
$$

In the given equation, $\alpha_{i}$ is a model parameter related to the internal length

$$
\ell_{i}:=\int_{0}^{L_{i} / 2}\left|\rho_{i}(\tau)\right| d \tau,
$$




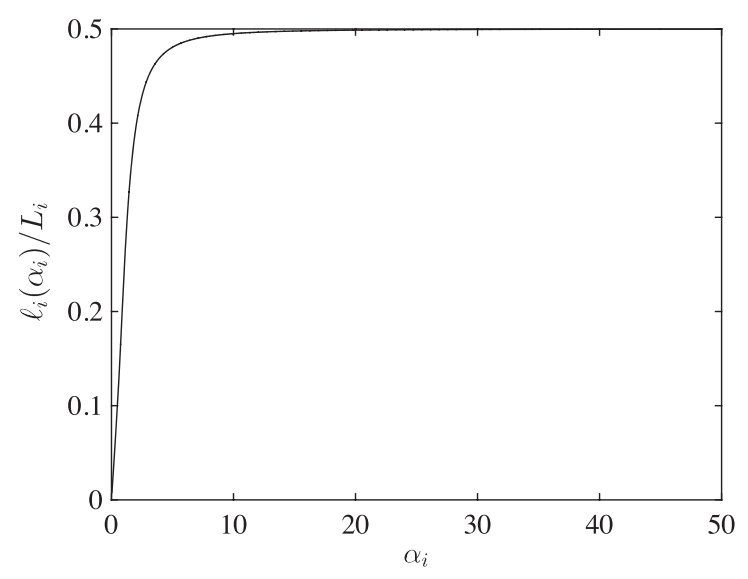

Figure 2. Graph of mapping $\alpha_{i} \mapsto \ell_{i}\left(\alpha_{i}\right) / L_{i}$ (Equation (3.5)).

which is interpreted - in the periodic setting under consideration - as the spatial correlation length of the Gaussian random field along $\boldsymbol{e}_{i}$. It can be shown, after little algebra, that $\alpha_{i}$ and $\ell_{i}$ are related through

$$
\ell_{i}=\frac{L_{i}}{2} \exp \left\{-\alpha_{i}^{-2}\right\} \mathcal{I}_{0}\left(\alpha_{i}^{-2}\right),
$$

with $\mathcal{I}_{0}$ the zero-order modified Bessel function. It should be noted that by construction, one has $\ell_{i}<L_{i} / 2$. The graph of the mapping $\alpha_{i} \mapsto \ell_{i}$ is depicted in Figure 2, which shows the fast rate of convergence of $\ell_{i}$ towards $L_{i} / 2$. The natural extension $\left\{\xi(\boldsymbol{y}), \boldsymbol{y} \in \mathbb{R}^{2}\right\}$ of the Gaussian field in $\mathbb{R}^{2}$ is then defined by imposing the periodicity condition

$$
\xi\left(y+L \diamond y^{\prime}\right)=\xi(y), \quad \forall y \in \Omega, \quad \forall y^{\prime} \in \mathbb{Z}^{2}
$$

almost surely, where $\boldsymbol{L}:=\left(L_{1}, L_{2}\right)$ and $\diamond$ denote the component-wise (Hadamard) product.

In a second stage, and upon substituting for simplicity the event $\left\{\bar{h}(\boldsymbol{y})>h_{0}^{\min }\right\}$ for the event $\left\{\bar{h}(\boldsymbol{y}) \geqslant h_{0}(\boldsymbol{x})\right\}$ in the consistency condition, the random field $\{\bar{h}(\boldsymbol{y}), \boldsymbol{y} \in \Omega\}$ is next defined through a nonlinear measurable mapping $\mathcal{T}$ such that

$$
\bar{h}(\boldsymbol{y}):=\mathcal{T}(\xi(\boldsymbol{y})), \quad \forall \boldsymbol{y} \in \Omega,
$$

where $\{\xi(y), y \in \Omega\}$ is the auxiliary Gaussian random field defined earlier and will be referred to as the Gaussian seed hereinafter. From a methodological standpoint, the mapping $\mathcal{T}$ is constructed such that $\{G(\boldsymbol{y}), \boldsymbol{y} \in \Omega\}$ exhibits a target family of first-order marginal probability distributions ensuring that

$$
\bar{h}(\boldsymbol{y})<h_{0}^{\min }, \quad \forall \boldsymbol{y} \in \Omega
$$

almost surely. This condition shows that the random field $\{\bar{h}(\boldsymbol{y}), \boldsymbol{y} \in \Omega\}$ defining the topography must be bounded from earlier almost surely. In the next section, we propose a random field model satisfying such a property. Note that this case specifically allows deep valleys (which may strongly impact the local flow) to be generated. Hence, because such valleys are likely to be filtered out in the multi-resolution analysis, this application is expected to be more critical for the coarse-scale simulations as compared with the case where a lower bound is also considered in (3.8).

3.1.2. Random field model. In order to ensure that the random field $\{\bar{h}(\boldsymbol{y}), \boldsymbol{y} \in \Omega\}$ is bounded from earlier, let us first introduce the algebraic decomposition

$$
\bar{h}(\boldsymbol{y}):=h_{0}^{\min }-G(\boldsymbol{y}), \quad \forall \boldsymbol{y} \in \Omega,
$$


where $\{G(\boldsymbol{y}), \boldsymbol{y} \in \Omega\}$ is an auxiliary non-Gaussian random field such that

$$
G(\boldsymbol{y})=\mathcal{H}(\xi(\boldsymbol{y})),
$$

in which $\mathcal{H}$ is a nonlinear mapping to be constructed. As $\{\bar{h}(\boldsymbol{y}), \boldsymbol{y} \in \Omega\}$ is centered (i.e., $\mathbb{E}\{\bar{h}(\boldsymbol{y})\}=0$ for all $\boldsymbol{y}$ in $\Omega$ ), it follows from Equation (3.9) that

$$
\mathbb{E}\{G(\boldsymbol{y})\}=h_{0}^{\min }, \quad \forall \boldsymbol{y} \in \Omega .
$$

The consistency condition further implies that

$$
G(y)>0, \quad \forall y \in \Omega,
$$

almost surely. Invoking information theory and the principle of maximum entropy [34, 35], it can be shown that the most objective candidate for the probability distribution of $G(\boldsymbol{y})$ (i.e., the one that reduces the modeling bias), $\boldsymbol{y}$ being fixed in $\Omega$, is then the Gamma distribution defined by shape parameter $s_{1}=1 / \delta^{2}$ and scale parameter $s_{2}=h_{0}^{\min } \delta^{2}$, with $\delta$ the coefficient of variation of $G(\boldsymbol{y})$ such that $0 \leqslant \delta<1 / \sqrt{2}$. As a consequence, Equation (3.10) now reads as

$$
G(\boldsymbol{y})=\left(F_{\mathcal{G}\left(s_{1}, s_{2}\right)}^{-1} \circ \Phi\right)(\xi(\boldsymbol{y})), \quad \forall \boldsymbol{y} \in \Omega,
$$

where $F_{\mathcal{G}\left(s_{1}, s_{2}\right)}^{-1}$ is the Gamma inverse cumulative distribution function with hyperparameters $\left(s_{1}, s_{2}\right)$, and $\Phi$ is inverse cumulative distribution function of the normalized Gaussian distribution. The random topography with Gamma first-order marginal distribution, which is specifically denoted by $\left\{\bar{h}_{\mathcal{G}}(\boldsymbol{y}), \boldsymbol{y} \in \Omega\right\}$ for later use, is subsequently defined as

$$
\bar{h}_{\mathcal{G}}(\boldsymbol{y}):=h_{0}^{\min }-\left(F_{\mathcal{G}\left(s_{1}, s_{2}\right)}^{-1} \circ \Phi\right)(\xi(\boldsymbol{y})), \quad \forall \boldsymbol{y} \in \Omega .
$$

This random field satisfies the constraints given by Equations (3.11) and (3.12), so that $\bar{h}(\boldsymbol{y})<h_{0}^{\min }$ almost surely for all $\boldsymbol{y}$ in $\Omega$. The proposed model depends on the following:

- The coefficient of variation $\delta$ controlling the statistical fluctuations of the field, with $0 \leqslant \delta<$ $1 / \sqrt{2}$

- The parameters involved in the definition of the periodic correlation function of the underlying Gaussian field $\{\xi(\boldsymbol{y}), \boldsymbol{y} \in \Omega\}$, namely, $\alpha_{1}$ and $\alpha_{2}$ of (3.3) in the present case.

The variance $\sigma_{\bar{h}}^{2}(\boldsymbol{y})$ of $\bar{h}(\boldsymbol{y})$ is seen to be independent of the spatial location $\boldsymbol{y} \in \Omega$ and is given by

$$
\sigma_{\bar{h}}^{2}(\boldsymbol{y})=\left(h_{0}^{\min }\right)^{2} \delta^{2},
$$

which allows one to scale the statistical fluctuations of $G(\boldsymbol{y})$ (which are specified by $\delta$ ), $\boldsymbol{y} \in \Omega$, given the macroscopic parameter $h_{0}^{\min }$ and a target variance for the random topography. In this work, the underlying Gaussian random field $\{\xi(\boldsymbol{y}), \boldsymbol{y} \in \Omega\}$ is sampled using a truncated Karhunen-Loève expansion, the order of which is classically determined by means of a convergence analysis on the ordered sequence of eigenvalues of the covariance kernel.

\subsection{Forward simulations at fine and coarse scales}

3.2.1. Multi-resolution analysis. The microscopic flow problem is described by a system of stochastic partial differential equations with highly oscillating coefficients, discretized over a fine grid with $N_{f 1}$ and $N_{f 2}$ elements along $\boldsymbol{e}_{1}$ and $\boldsymbol{e}_{2}$, respectively. In practice, and in addition to standard pathwise convergence criteria, the number of finite elements in a given direction is determined such that the correlation structure of $\{\bar{h}(\boldsymbol{y}), \boldsymbol{y} \in \Omega\}$ is properly caught. This is typically achieved by imposing a selected number of integration points, say six or eight, per spatial correlation length. When this meshing rule leads to different values of $N_{f 1}$ and $N_{f 2}$, the microscopic mesh is defined using $N_{f}:=\max \left(N_{f 1}, N_{f 2}\right)$ elements along each direction. The macroscopic flow problem is then seen as a coarse-scale approximation defined by smoother matrix-valued coefficients $\{[\boldsymbol{A}(\boldsymbol{x})], \boldsymbol{x} \in \Omega\}$ 


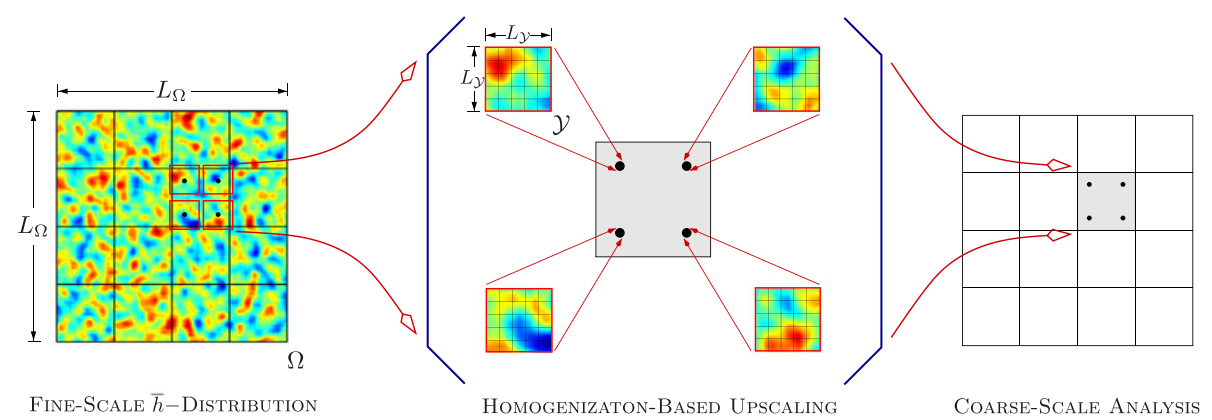

Figure 3. The multi-resolution analysis of Section 3.2.1 is depicted. For a given fine-scale roughness distribution, the macroscopic domain $\Omega$ is first assigned a coarse mesh. Next, around each integration point of this mesh, a local domain $\mathcal{Y}$ is chosen. These domains may or may not overlap. Homogenization relations (2.8) then associate macroscopic tensorial coefficients with each integration point, which can then be used to compute a coarse-scale solution.

and $\{[\boldsymbol{C}(\boldsymbol{x})], \boldsymbol{x} \in \Omega$ \}, defined by means of a local upscaling. In accordance with the microscopic mesh, the associated macroscopic mesh is defined by considering $N_{c}$ elements in each direction of the canonical basis. The refinement ratio $\eta:=N_{f} / N_{c}$, which could be made direction-dependent, hence defines a multi-resolution analysis that is algorithmically reminiscent of the classical two-scale homogenization setup. Specifically, at each macroscopic point $\boldsymbol{x}$, the coefficients $[\boldsymbol{A}(\boldsymbol{x})]$ and $[\boldsymbol{C}(\boldsymbol{x})]$ are computed on domain $\mathcal{Y}=\left[x_{1}-L_{\mathcal{Y}} / 2, x_{1}+L_{\mathcal{Y}} / 2\right] \times\left[x_{2}-L_{\mathcal{Y}} / 2, x_{2}+L_{\mathcal{Y}} / 2\right]$ using the classical two-scale results (2.8), with

$$
L_{\mathcal{Y}}=R \times \frac{L_{\Omega}}{N_{f}} .
$$

This numerical scheme is depicted in Figure 3. The parameter $R \in \mathbb{N}_{*}$ therefore controls how smallscale information is filtered through the homogenization:

- If taken too small, the random fields $\{[\boldsymbol{A}(\boldsymbol{x})], \boldsymbol{x} \in \Omega\}$ and $\{[\boldsymbol{C}(\boldsymbol{x})], \boldsymbol{x} \in \Omega\}$ are still highly oscillating, and the two-scale approach loses its very purpose;

- If too large (in the sense that $R \gg \max \left(\left\{\ell_{i}\right\}_{i=1}^{2}\right.$ ), oscillations may be totally smoothed out, and the macroscopic solution becomes deterministic and insensitive to small-scale features.

In practice, appropriate choices for parameters $\eta$ and $R$ are guided by the prediction of a given quantity of interest (QoI), such as peak pressure or load capacity, as well as by the selection of a given validation criterion.

3.2.2. Illustrative results in $1 D$. In this section, the capabilities of the multi-resolution approach will first be demonstrated in the one-dimensional setting where the solution features can be easily observed. While the emphasis is not on the particular choice of numerical parameters here - but rather on how these parameters influence the analysis outcome - the selected correlation lengths are purposely chosen such that $\ell \ll L_{\Omega}$. The case where the scale separation is less pronounced will be illustrated through 2D computations in Section 3.2.3. In all cases, the surface model of Section 3.1.2 will be employed. For 1D examples, $\sigma_{\bar{h}}=0.09$ is chosen in (3.15). The units of position $(\mu \mathrm{m})$ and pressure (MPa) will not be explicitly indicated in the figures.

Probability density functions of the peak pressure for different correlation lengths $\ell_{1}=\ell$ and representative pressure distributions are provided in Figure 4 for both fine-scale and coarse-scale analysis approaches discussed so far. For the homogeneous interface, there is a single well-defined pressure distribution. For the fine-scale analysis of the heterogeneous interface, the pressure distribution and hence the peak pressure depend on the particular realization of the interface (Figure 4(a-1)). This is an example to how local roughness features can significantly influence the solution even for a correlation length that is as small as $L_{\Omega} / 300$, as previously noted in Section 2.2. By contrast, it may 


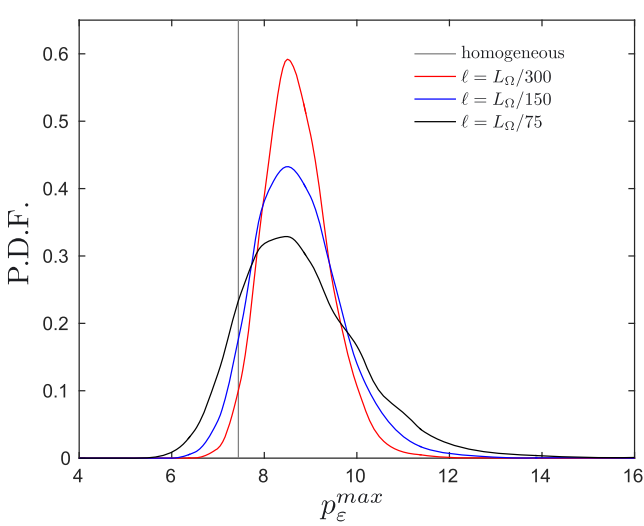

(a-1) peak pressure for fine-scale analysis

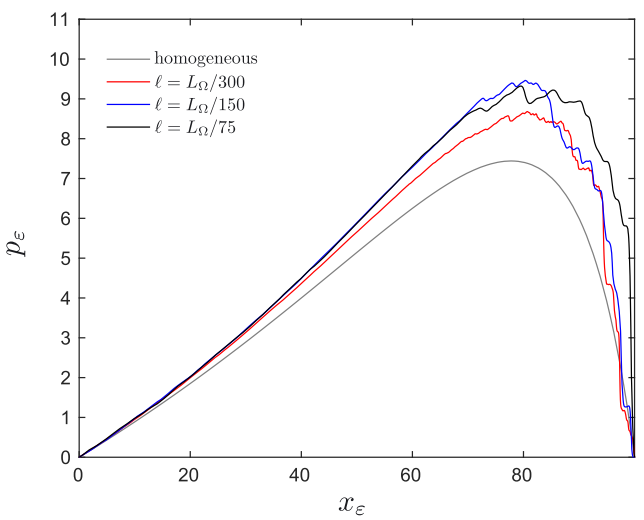

(b-1) pressure distribution for fine-scale analysis

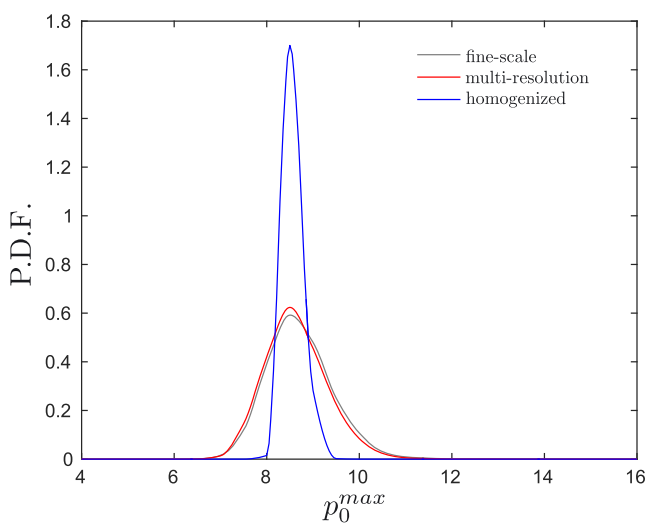

(a-2) peak pressure for coarse-scale analysis

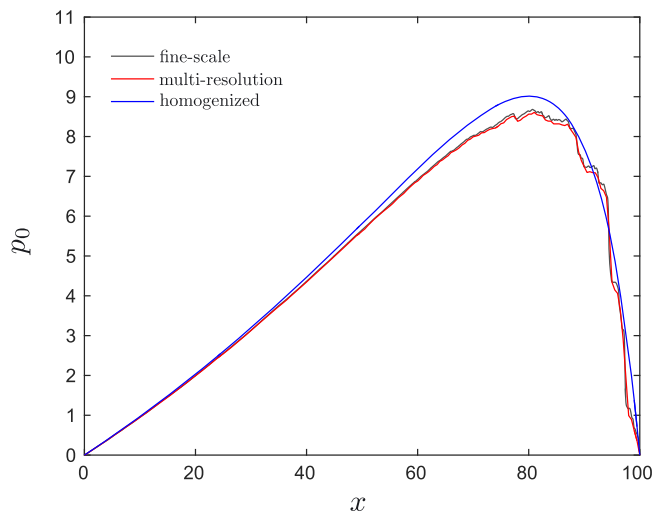

(b-2) pressure distribution for coarse-scale analysis

Figure 4. The probability density functions of the peak pressure associated with (a-1) 1D fine-scale analyses at different correlation lengths $\ell_{1}=\ell$ with $N_{f}=2000$ and (a-2) the coarse-scale approaches with $N_{c}=200$ are shown, both based on $10^{4}$ realizations with $\sigma_{\bar{h}}=0.09 \mu \mathrm{m}$. The latter consist of the multi-resolution approach corresponding to $\ell=L_{\Omega} / 300$ using $R=20$ and the classical homogenization scheme with the same microstructure at each macroscopic integration point. Selected pressure distributions across the interface for these cases are depicted in (b-1) and (b-2) where the homogeneous and fine-scale solutions, respectively, are also displayed for reference.

be noted here that for a periodic surface roughness with a sinusoidal profile, scale separation would already be clearly observed at a roughness period of $L_{\Omega} / 200$. The fact that the absolute length scale also plays a role, in view of its effect on the probability distribution, is an additional indication that scale separation does not hold for this random interface. Note that roughness delivers a higher mean peak pressure than a smooth interface in all cases.

Now, the coarse-scale analysis of the interface may be carried out in a number of ways (Figure 4(a2)). First, in the context of Section 2.2, one may attach a relatively large random sample to each macroscopic mesh integration point. The size is chosen presently as $L y=6 \ell$, the absolute value of $\ell$ being irrelevant for homogenization. Because the corresponding probability distribution is still rather spread suggests that an even larger sample size is required. Note, however, that this distribution is already significantly more peaked than the ones for the fine-scale, and larger sample sizes only make the distribution more peaked, consistent with the fact that the homogenization approach assumes scale separation. The multi-resolution analysis, on the other hand, makes direct use of the underlying fine-scale microstructure and hence displays a distribution that is much closer to its corresponding distribution from the fine-scale analysis. Hence, despite the fact that the multi-resolution analysis relies on homogenization theory for upscaling, it clearly performs significantly better towards the 
estimation of the spread in the macroscopic response for the present case where there is no strict scale separation. The mean peak pressure estimations are close for all approaches.

The pressure distributions across the interface also reflect some of these observations. Figure 4(b1) summarizes selected fine-scale solutions, displaying the smoother variations with increasing correlation length. For the coarse-scale solutions, the multi-resolution analysis can deliver a distribution that is very close to the fine-scale solution, reflecting the significant influence of local microstructural features in a similar manner (Figure 4(b-2)). Classical homogenization, by construction, delivers a smooth curve, which, in view of the corresponding probability distribution, depends only slightly on the particular realization at the chosen sample size $\left(L_{y}=6 \ell\right)$.

The size $L y$ of the upscaling domain in the multi-resolution approach, determined by $R$ in (3.16), controls the quality of the coarse-scale solution. For the parameters of Figure 4, the coarse-scale solution is compared against the fine-scale solution for two different realizations with $\ell=L_{\Omega} / 300$ in Figure 5 for increasing values of $R$, all at a resolution of $N_{c}=200$. Too small values do not correctly reflect the local microstructural features while too large values can lead to significant smoothing, as indicated in Section 3.2.1. Note that it is desirable to choose this value as small as possible in order to minimize the cost of upscaling computations. Presently, $R=20$ appears to lie in an optimal range. The optimal value of $R$ will depend on the numerical parameters of the problem, which is also the case for $N_{c}$. For simplicity, keeping $R=20$ constant, $N_{c}$ is varied in Figure 6 within neighborhoods of 100,200 , and 300 (by incrementing it by 1 within \pm 10 ) while the underlying fine-scale roughness

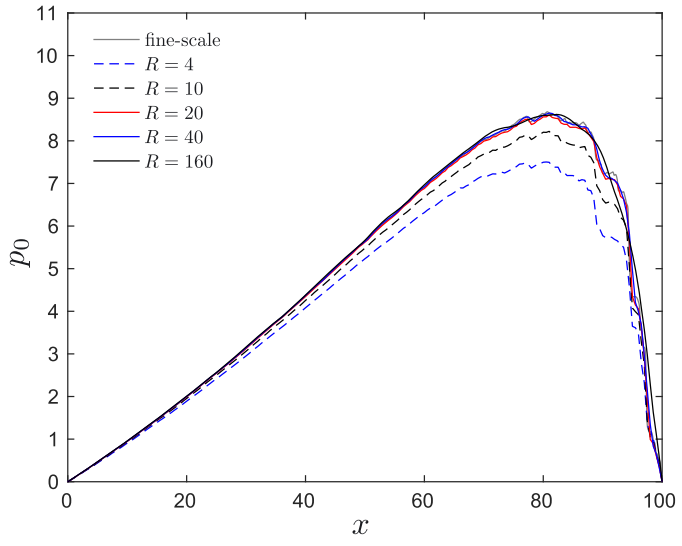

(a) realization 1

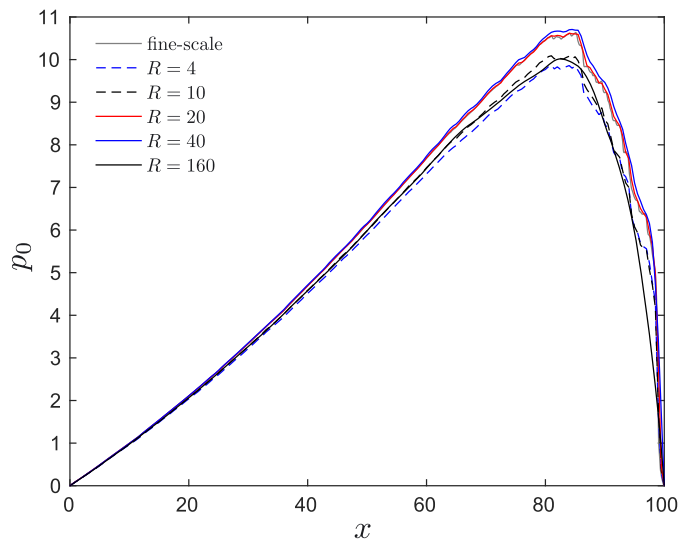

(b) realization 2

Figure 5. For two different realizations of the interface with $\ell=L_{\Omega} / 300$ ), the multi-resolution coarse-scale solution is computed with $N_{c}=200$ at different values of $R$ from (3.16). Realization 1 is borrowed from Figure 4. Fine-scale computation employs $N_{f}=2000$ elements.

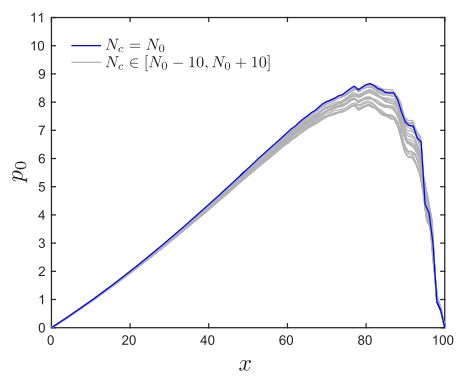

(a) $N_{0}=100$

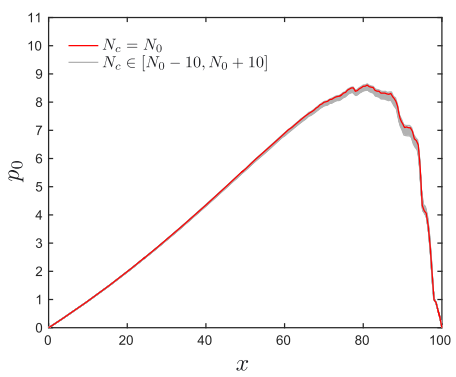

(b) $N_{0}=200$

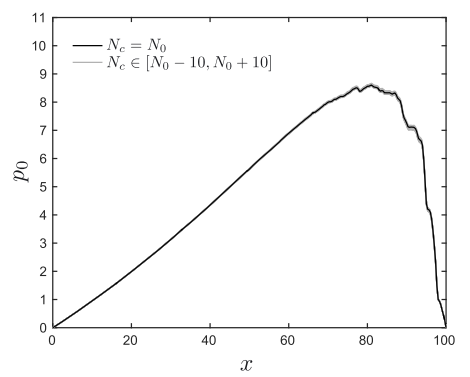

(c) $N_{0}=300$

Figure 6. The coarse-scale mesh sensitivity of the multi-resolution solution is assessed using realization 1 of Figure 5 by first assigning an initial value $N_{c}=N_{0}$ and then computing 20 additional solutions by varying $N_{c}$ within an interval of \pm 10 elements about this default value. In all cases, $R=20$. 


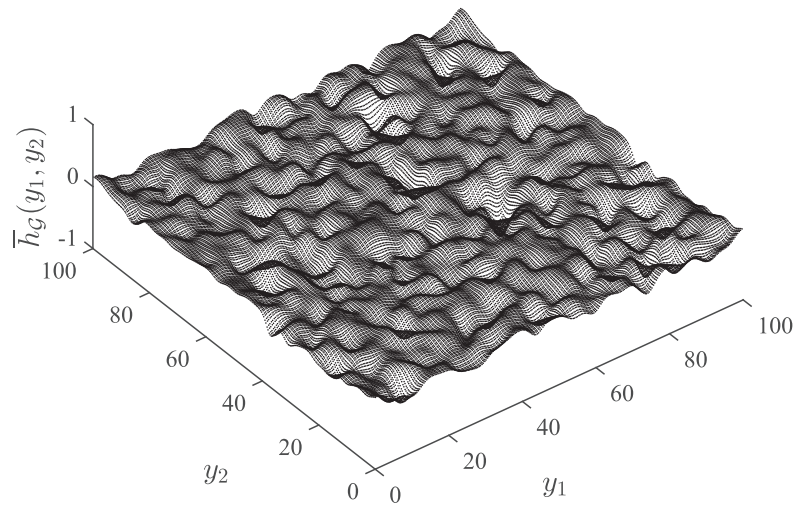

Figure 7. One realization of the random topography $\left\{\bar{h}_{\mathcal{G}}(\boldsymbol{y}), \boldsymbol{y} \in \Omega\right\}$, defined with $\ell_{1}=\ell_{2}=3$.

is kept the same as in Figure 5(a). Clearly, $N_{c}=100$ is not a suitable choice because the coarse-scale solution is highly mesh sensitive. This sensitivity is already eliminated significantly at $N_{c}=200$.

3.2.3. Illustrative results in $2 D$. One realization of the random field $\left\{\bar{h}_{\mathcal{G}}(\boldsymbol{y}), \boldsymbol{y} \in \Omega\right\}$, defined with $\ell_{1}=\ell_{2}$ and $\delta=0.45$ (hence, $\sigma_{\bar{h}}=0.09$ ), is shown in Figure 7. A representative two-dimensional result is additionally presented in Figure 8, for one realization of the random topography. Here, it can also again be observed (by comparing Figure 8(a-1) with Figure 8(a-2)) that it is important to resolve roughness effects because it leads to a higher peak pressure compared with the homogeneous interface. Even larger differences can easily be observed if surface roughness is increased further by increasing $\sigma_{\bar{h}}$, subject to the restriction $0 \leqslant \delta<1 / \sqrt{2}$ (Section 3.1.2) via (3.15). As in the one-dimensional example, the multi-resolution approach can well represent the highly oscillatory fine-scale solution and deliver a peak pressure of comparable magnitude. For a resolution that is close to the fine scale computation, the statistical information is highly localized, so that selecting a large mesoscale domain (by increasing $R$ ) generates a (nonlocal) bias. Conversely, coarser resolutions impose retaining a larger value of $R$ in order to account for local subscale features in the macroscopic response. Finally, it is remarked that negative pressure values (with respect to the boundary values) can be observed in one- and two-dimensional settings, depending on the roughness distribution near $h_{0}^{\min }$. Physically, these may indicate regions where cavitation needs to be taken into account. Presently, cavitation is not addressed.

The influence of both $\eta$ and $R$ on the probability distribution of the peak pressure $p_{0}^{\max }$ is investigated in Figure 9. As expected, it is seen that selecting a coarser resolution shifts the probability density function of the peak pressure, no matter the value of $R$. Moreover, the mean value of the peak pressure is all the more underestimated than $\eta$ is large, while its statistical fluctuations are seen to decrease with $\eta$ and to weakly depend on $R$ for a given value of $\eta$. The relative error between the reference mean value, obtained from the fine-scale computations, and the coarse-scale approximations with $\eta=5$ is equal to $23.1 \%, 26.5 \%, 28.8 \%$, and $30.3 \%$ for $R=5, R=10, R=15$, and $R=20$ respectively. The plot of the mean pressure field is further shown in Figure 10 for the reference fine-scale simulation and several coarse-scale computations defined by $\eta=10$.

Whereas the mean value of $p_{0}^{\max }$ was found to be almost insensitive to the choice of $R$ for a given coarse resolution, it can be observed that increasing the size of the homogenization domain $\mathcal{Y}$ allows for a better prediction of the mean field. In order to quantify the error introduced on the mean field more precisely, we then introduce the mapping $\mathcal{E}$ that measures the relative error in the $L^{2}$ norm of the mean solution field, as a function of $R$ (here, the value of $\eta$ is assumed to be fixed):

$$
\mathcal{E}(R)=\left(\| \mathbb{E}\left\{p_{0}(\boldsymbol{x}\}\|-\| \mathbb{E}\left\{p_{\epsilon}(\boldsymbol{y}\} \|\right) / \| \mathbb{E}\left\{p_{\epsilon}(\boldsymbol{y}\} \|,\right.\right.\right.
$$




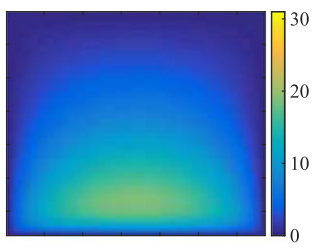

(a-1) Homogeneous case

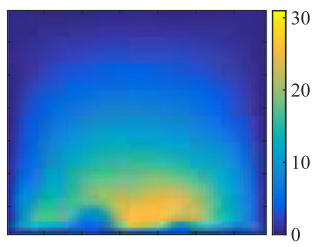

(b-2) $\eta=2, R=10$

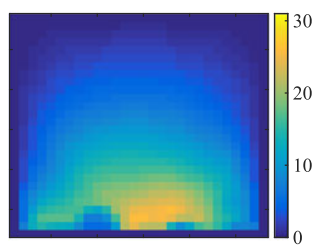

(c-2) $\eta=5, R=10$

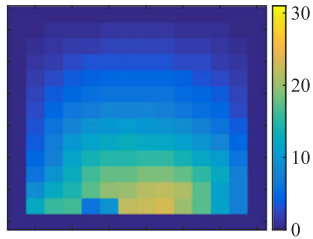

(d-2) $\eta=10, R=10$

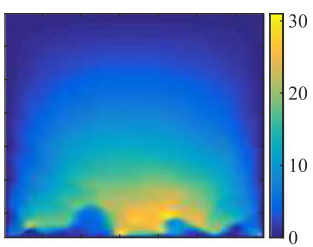

(a-2) Fine-scale result

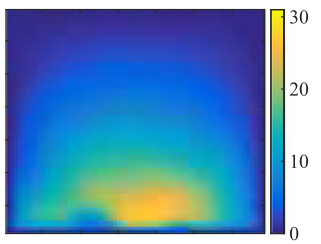

(b-3) $\eta=2, R=15$

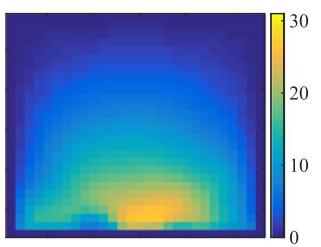

(c-3) $\eta=5, R=15$

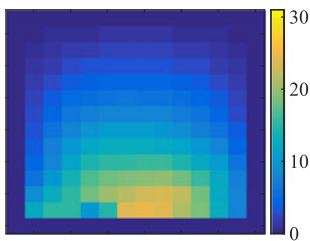

(d-3) $\eta=10, R=15$

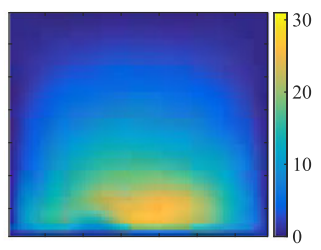

(b-4) $\eta=2, R=20$

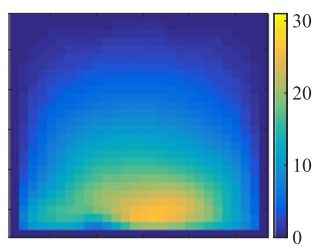

(c-4) $\eta=5, R=20$

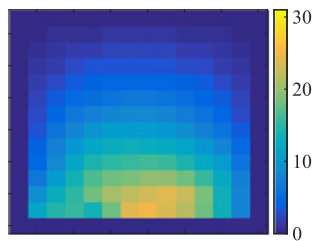

(d-4) $\eta=10, R=20$

(d-1) $\eta=10, R=5$

Figure 8. The multi-resolution approach is demonstrated on the pressure solution in $2 \mathrm{D}$, using one realization of the random topography $\left\{\bar{h}_{\mathcal{G}}(\boldsymbol{y}), \boldsymbol{y} \in \Omega\right\}$ with $\ell_{1}=\ell_{2}=3\left(L_{\Omega}=100\right), \eta \in\{2,5,10\}$ (from top to bottom) and $R \in\{5,10,15,20\}$ (from left to right). The homogeneous case and the fine-scale simulation are depicted at the very top of the figure.

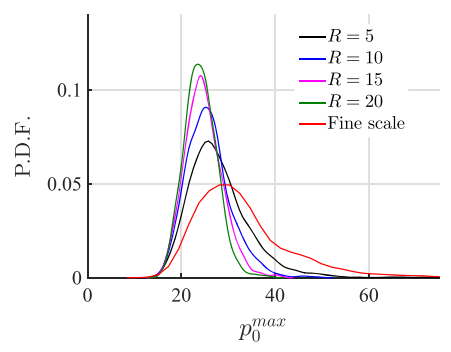

(a) $\eta=2$

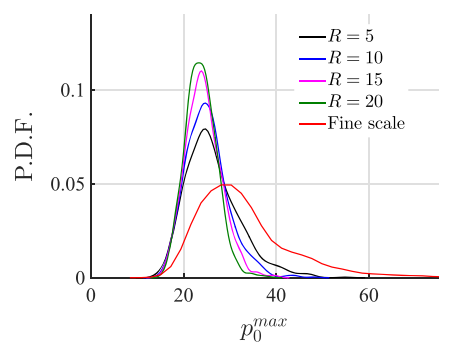

(b) $\eta=5$

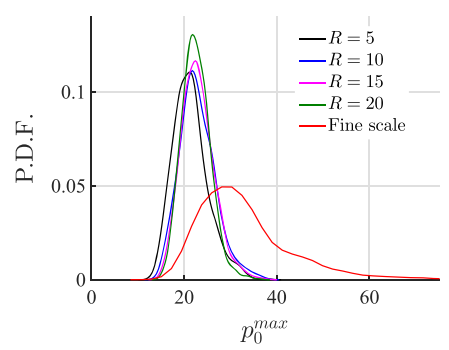

(c) $\eta=10$

Figure 9. The probability density function of the peak pressure $p_{0}^{\max }$ is shown for three coarse-scale resolutions, with $\eta \in\{2,5,10\}$, and different sizes of the homogenization domain $\mathcal{Y}$.

where $p_{0}$ implicitly depends on $R$, and

$$
\|f(z)\|=\left(\int_{\Omega} f(z)^{2} \mathrm{~d} z\right)^{1 / 2} .
$$




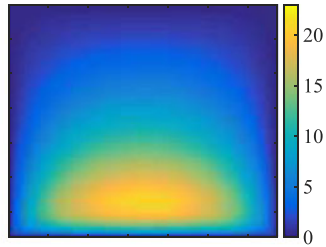

(a) Fine-scale result

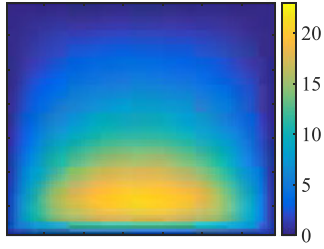

(b-1) $\eta=2, R=5$

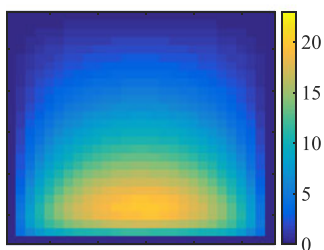

(c-1) $\eta=5, R=5$

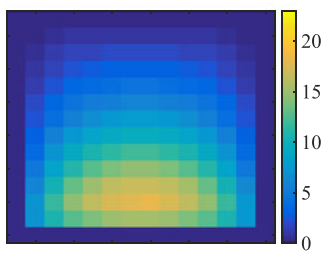

(d-1) $\eta=10, R=5$

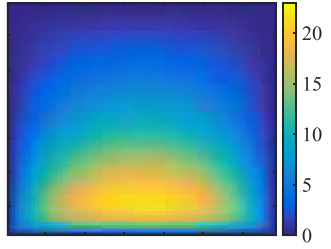

(b-2) $\eta=2, R=10$

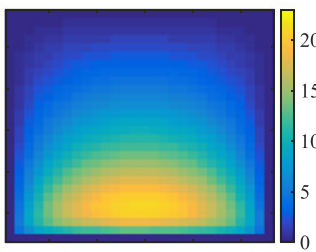

(c-2) $\eta=5, R=10$

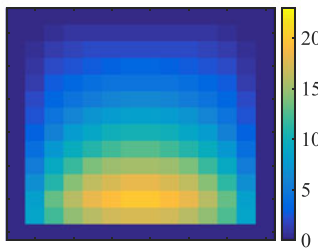

(d-2) $\eta=10, R=10$

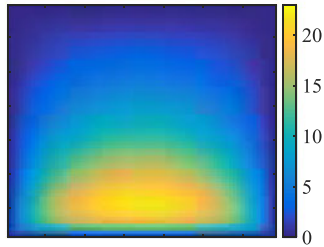

(b-3) $\eta=2, R=15$

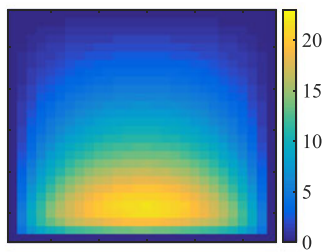

(c-3) $\eta=5, R=15$

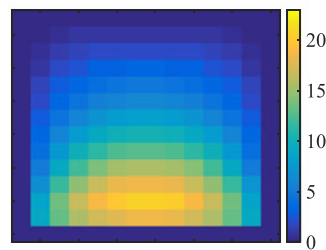

(d-3) $\eta=10, R=15$

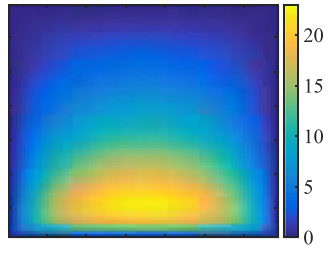

(b-4) $\eta=2, R=20$

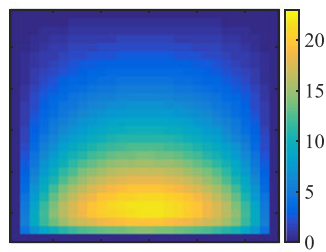

(c-4) $\eta=5, R=20$

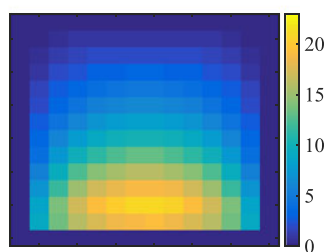

(d-4) $\eta=10, R=20$

Figure 10. Evolution of the mean pressure field across the scales. The fine-scale result is depicted in (a), while the coarse-scale estimates are represented in (b), (c), and (d) for $\eta=2,5$, and 10, respectively.

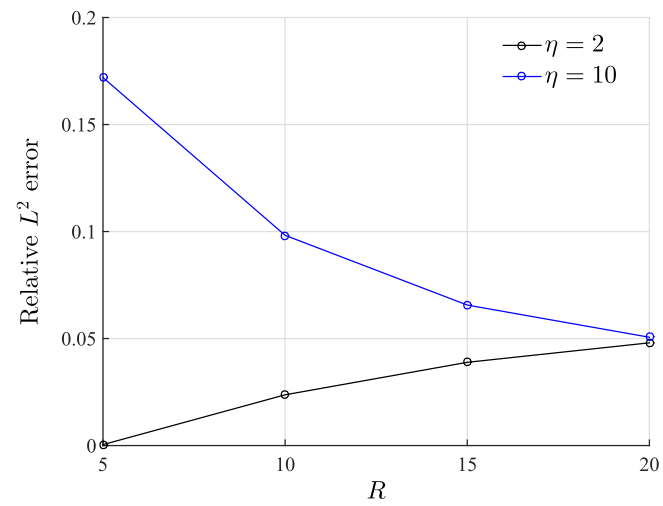

(a) Relative $L^{2}$ error in mean (graph of $R \mapsto \mathcal{E}(R)$ )

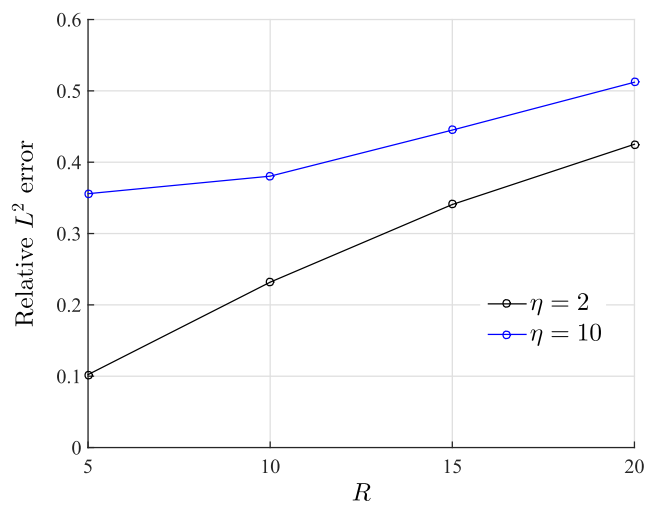

(b) Relative $L^{2}$ error in standard deviation

Figure 11. For $\eta=10$, the relative errors in the $L^{2}$ norm of the mean (3.17) and standard deviation fields are computed for increasing values of $R$. 
The convergence of this relative error is depicted in Figure 11 (with $\eta=10$ ), where a similar error for the field of standard deviation is also shown.

Because of the localization of information induced by the local upscaling, it is seen that the mean field is better approximated at large $R$ for very coarse representations, while increasing $R$ at resolutions that are close to the finest scale adds more bias in the field of mean pressure.

\subsection{Coarse-scale stochastic modeling}

In what follows, the notation $\left[\operatorname{diag}\left(A_{11}, \ldots, A_{n n}\right)\right]$ denotes the $(n \times n)$ diagonal matrix with entries $A_{11}, \ldots, A_{n n}$. In addition, $c(\boldsymbol{x})$ stands for the general notation of the normalization constant involved in a given probability density function at point $\boldsymbol{x}$ - the value of $c(\boldsymbol{x})$ may therefore change from line to line. Finally, $\left[I_{n}\right]$ is the identity matrix of size $n$.

3.3.1. Statistical characterization of the matrix-valued coefficients. For $\delta=0.45, \eta=10$, and $R=10$, the graphs of the mappings $\boldsymbol{x} \mapsto\|[\underline{A}(\boldsymbol{x})]\|$ and $\boldsymbol{x} \mapsto\|[\underline{C}(\boldsymbol{x})]\|$ related to the mean fields of the matrix-valued coefficients are shown in Figure 12, while the graphs of the coefficients of variation

$$
\delta_{[A]}(\boldsymbol{x}):=\left(\frac{\mathbb{E}\left\{\|[\boldsymbol{A}(\boldsymbol{x})]-[\underline{A}(\boldsymbol{x})]\|^{2}\right\}}{\|[\underline{A}(\boldsymbol{x})]\|^{2}}\right)^{1 / 2} \text { and } \delta_{[\boldsymbol{C}]}(\boldsymbol{x}):=\left(\frac{\mathbb{E}\left\{\|[\boldsymbol{C}(\boldsymbol{x})]-[\underline{C}(\boldsymbol{x})]\|^{2}\right\}}{\|[\underline{C}(\boldsymbol{x})]\|^{2}}\right)^{1 / 2}
$$

are shown in Figure 13 (note that these graphs involve statistical estimators of these quantities, obtained with 1000 independent realizations of the random topography and that no specific notation is introduced to denote these estimators, by simplicity).

These figures clearly illustrate the spatial variations of the mean and dispersion fields, which indicates a non-stationarity for the homogenized coefficients $\{[\boldsymbol{A}(\boldsymbol{x})], \boldsymbol{x} \in \Omega\}$ and $\{[\boldsymbol{C}(\boldsymbol{x})], \boldsymbol{x} \in \Omega\}$. Moreover, it is observed that the random field $\{[A(x)], x \in \Omega\}$ exhibits much larger fluctuations than $\{[\boldsymbol{C}(\boldsymbol{x})], \boldsymbol{x} \in \Omega\}$, which may be explained by a stronger dependence on the third power of $h$ in view of (2.8). It should be noticed that the dispersion fields present some noisy oscillations (at large fluctuations, for $x_{2} \rightarrow L_{2}$ ) that are induced by a slight undersampling.

While the random variable $[\boldsymbol{A}(\boldsymbol{x})], \boldsymbol{x} \in \Omega$, is symmetric almost surely, the variable $\{[\boldsymbol{C}(\boldsymbol{x})]$ can theoretically be asymmetric. However, computational evidence shows that the exhibited level of asymmetry is generally very small. In the present study, the global level of asymmetry is characterized by the random variable

$$
\varepsilon^{s}:=\max _{\boldsymbol{x} \in \mathcal{D}(\Omega)} \frac{1}{2} \frac{\left\|[\boldsymbol{C}(\boldsymbol{x})]^{\mathrm{T}}-[\boldsymbol{C}(\boldsymbol{x})]\right\|}{\|[\boldsymbol{C}(\boldsymbol{x})]\|},
$$

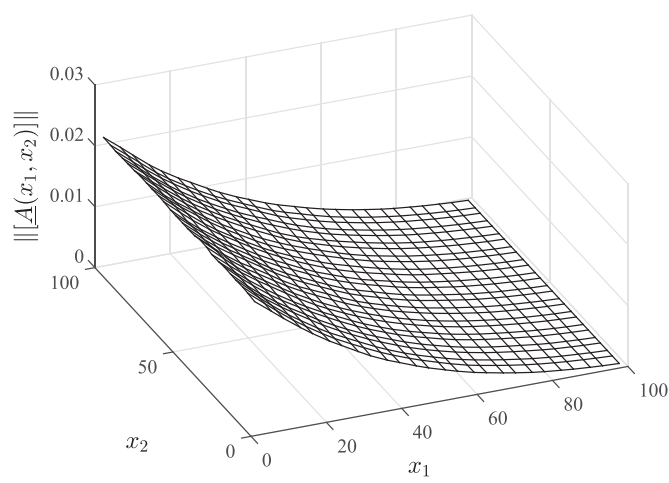

(a)

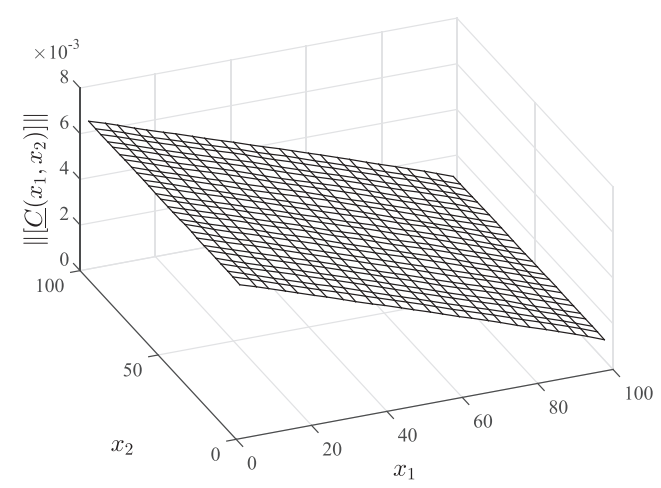

(b)

Figure 12. For $\eta=10$ and $R=10$, the mean functions of the random fields $\{[\boldsymbol{A}(\boldsymbol{x})], \boldsymbol{x} \in \Omega\}$ and $\{[\boldsymbol{C}(\boldsymbol{x})]$, $x \in \Omega\}$ (which correspond to the homogenized coefficients in the governing equation at the macroscale), are shown and illustrate the non-stationarity of these fields. 


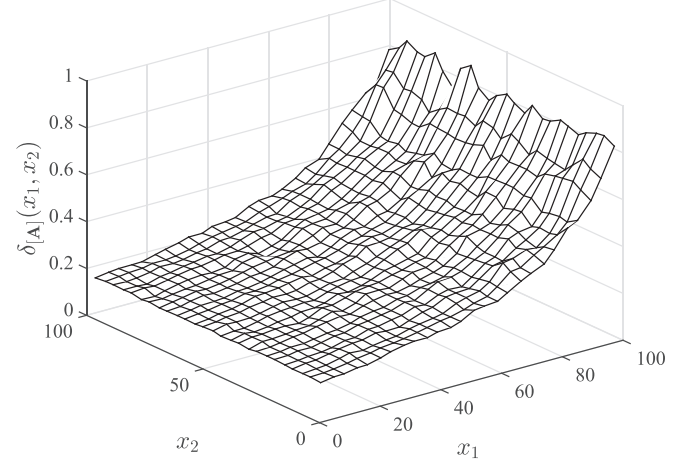

(a)

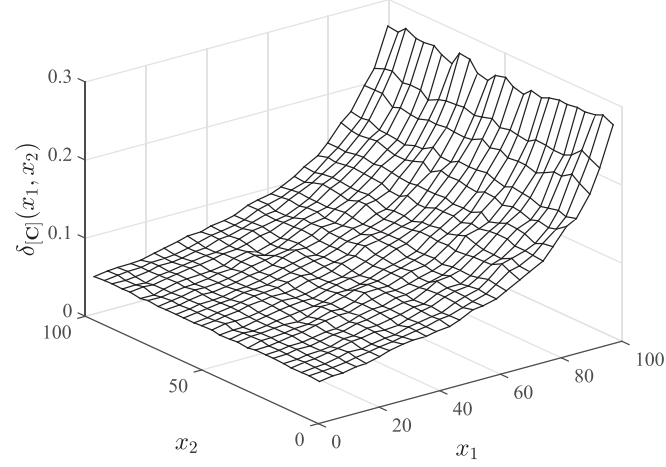

(b)

Figure 13. For $\eta=10$ and $R=10$, the functions characterizing the fluctuations of the random fields $\{[\boldsymbol{A}(\boldsymbol{x})], \boldsymbol{x} \in \Omega\}$ and $\{[\boldsymbol{C}(\boldsymbol{x})], \boldsymbol{x} \in \Omega\}(3.19)$ are shown.

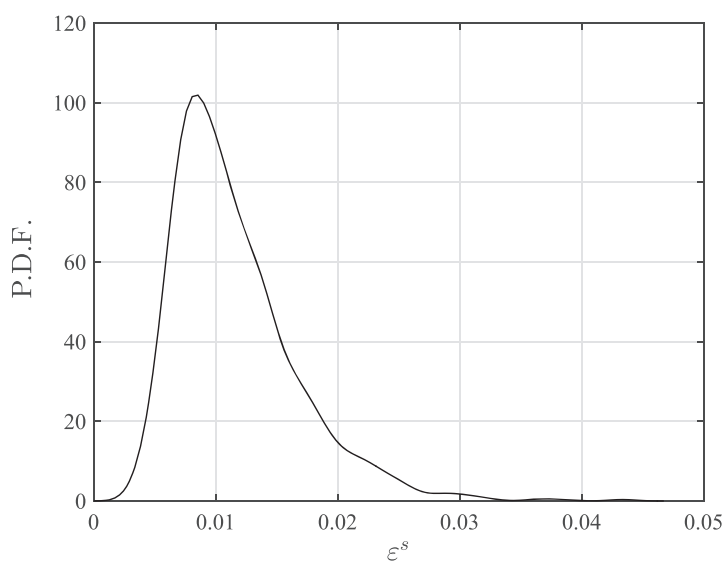

Figure 14. This figure shows the probability density function associated with random variable $\varepsilon^{s}$.

where $\mathcal{D}(\Omega)$ denotes the countable set of points involved in the macroscopic discretization of $\Omega$. The probability density function of $\varepsilon^{s}$ is shown in Figure 14. The mean value and the 99th percentile of $\varepsilon^{s}$ are respectively equal to $1.16 \%$ and $2.83 \%$, showing that the macroscopic coefficient $[\boldsymbol{C}(\boldsymbol{x})]$ is weakly asymmetric over $\Omega$ and can be approximated, for all $x$ fixed in $\Omega$, by a random variable with values in the set of symmetric positive-definite $(2 \times 2)$ matrices.

In what follows, the stochastic macroscopic coefficients will therefore be modeled as random fields with values in the set $\mathbb{M}_{2}^{+}(\mathbb{R})$ of symmetric positive-definite $(2 \times 2)$ real matrices. In order to expose the methodological aspects with simplified notations, let $\{[\boldsymbol{K}(\boldsymbol{x})], \boldsymbol{x} \in \Omega\}$ denote any of these two matrix-valued random fields. The structure of the macroscopic coefficients can be further investigated by characterizing the magnitude of the off-diagonal components - which may become negligible whenever the underlying topography exhibits, as presently, an isotropic correlation structure. To this aim, we introduce the relative error $\varepsilon_{[\boldsymbol{K}]}^{d}$ between $\{[\boldsymbol{K}(\boldsymbol{x})], \boldsymbol{x} \in \Omega\}$ and its diagonal approximation

$$
\varepsilon_{[\boldsymbol{K}]}^{d}:=\max _{\boldsymbol{x} \in \mathcal{D}(\Omega)} \frac{\left\|\left[\operatorname{diag}\left(K_{11}(\boldsymbol{x}), K_{22}(\boldsymbol{x})\right)\right]-[\boldsymbol{K}(\boldsymbol{x})]\right\|}{\|[\boldsymbol{K}(\boldsymbol{x})]\|},
$$

where

$$
\left[\operatorname{diag}\left(K_{11}(\boldsymbol{x}), K_{22}(\boldsymbol{x})\right)\right]:=\left[\begin{array}{cc}
K_{11}(\boldsymbol{x}) & 0 \\
0 & K_{22}(\boldsymbol{x})
\end{array}\right]
$$




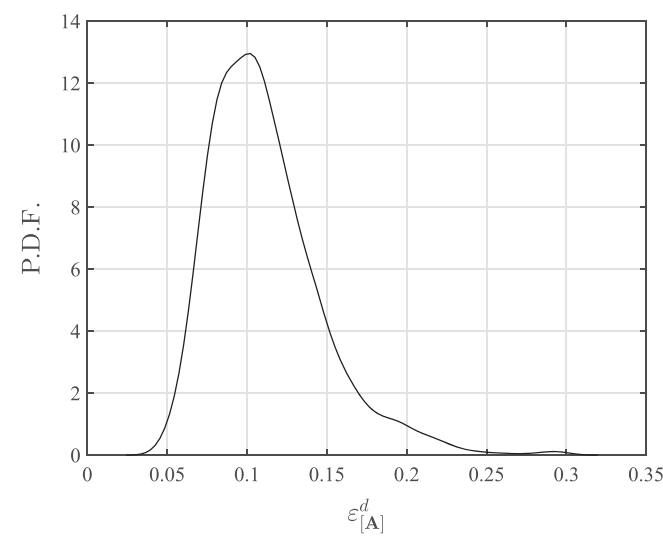

(a) P.D.F. of $\varepsilon_{[\boldsymbol{A}]}^{d}$

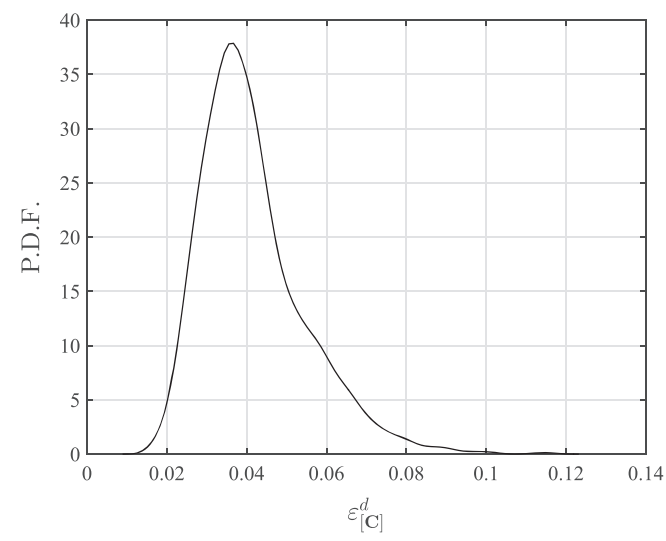

(b) P.D.F. of $\varepsilon_{[\boldsymbol{C}]}^{d}$

Figure 15. In this figure, the probability density functions of $\varepsilon_{[A]}^{d}$ and $\varepsilon_{[C]}^{d}$ are shown.

is the closest diagonal approximation of $[\boldsymbol{K}(\boldsymbol{x})]$ in the sense of the Euclidean projection. The probability density functions of $\varepsilon_{[A]}^{d}$ and $\varepsilon_{[C]}^{d}$ are depicted below in Figure 15. The mean values of $\varepsilon_{[A]}^{d}$ and $\varepsilon_{[C]}^{d}$ are respectively equal to $11.15 \%$ and $4.15 \%$. Consequently, neglecting the off-diagonal terms in the probabilistic modeling may yield a bias, which must be thoroughly quantified for some QoI. This point will be discussed in the following.

3.3.2. Methodology. Similarly to Section 3.1.1, and following some derivations proposed elsewhere in computational solid mechanics [36, 37], a probabilistic model for the random field $\{[\boldsymbol{K}(\boldsymbol{x})], \boldsymbol{x} \in$ $\Omega$ \} is sought through a point-wise memoryless transformation of an underlying normalized vectorvalued Gaussian field (with statistically independent components $\left\{\xi_{i}(\boldsymbol{x}), \boldsymbol{x} \in \Omega\right\}, 1 \leqslant i \leqslant q$, with $q \leqslant 3)$ :

$$
[K(x)]:=\mathcal{T}_{[K]}(\Xi(x), x), \quad \forall x \in \Omega .
$$

Note that the previous transformation depends on $x$ in view of the non-stationarity of the field. In effect, the nonlinear mapping $\mathcal{T}_{[K]}$ is explicitly constructed by prescribing the family $\left\{p_{[K(x)]}\right\}_{x \in \Omega}$ of first-order marginal distribution of the field and implicitly defines the whole system of marginal distributions of $\{[\boldsymbol{K}(\boldsymbol{x})], \boldsymbol{x} \in \Omega\}$ through (3.23). The construction of $\mathcal{T}_{[\boldsymbol{K}]}$ is achieved by defining $p_{[K(x)]}, \boldsymbol{x}$ being fixed, by means of the principle of maximum entropy. The latter is specifically invoked under the constraints [37]

$$
\mathbb{E}\{[K(\boldsymbol{x})]\}=[\underline{K}(\boldsymbol{x})], \quad \forall \boldsymbol{x} \in \Omega,
$$

and

$$
\mathbb{E}\{\log (\operatorname{det}([\boldsymbol{K}(\boldsymbol{x})]))\}=s(\boldsymbol{x}),|s(\boldsymbol{x})|<+\infty, \quad \forall \boldsymbol{x} \in \Omega .
$$

By definition, the support of $p_{[K(x)]}$ is $\mathbb{M}_{2}^{+}(\mathbb{R})$. The first condition given is related to the mean field $\boldsymbol{x} \mapsto[\underline{K}(\boldsymbol{x})]$, whereas the second one, together with a mild assumption related to the level of statistical fluctuations (see (3.42) for instance), allows one to prove that both $[\boldsymbol{K}(\boldsymbol{x})]$ and $[\boldsymbol{K}(\boldsymbol{x})]^{-1}$ are secondorder random variables.

\section{Remark 1}

In this work, the modeling approach for the matrix-valued random fields is based on informationtheoretic algebraic decompositions. By construction, such expansions are consistent with theoretical requirements (such as positive-definiteness) almost surely and typically rely on a low-dimensional parametrization that makes the calibration of the model hyperparameters more tractable. In addition, 
efficient sampling strategies were proposed in the literature, for both memoryless mappings [37] and transformations based on stochastic differential equations [38]. One limitation of such models is that they cannot exactly represent any second-order random field (in other words, each class of information-theoretic models defines a subset of the set of all second-order random fields), and more general representations relying on polynomial chaos expansions can be used to circumvent this potential drawback (see the discussion in [39]). From the perspective of uncertainty propagation, a Monte Carlo approach is presently used as the stochastic solver - survey on alternative approaches, together with other applications in computational mechanics, can be found in [40-43], to name a few. Finally, it should be noticed that a spectral approach involving a polynomial chaos expansion of the underlying random surface could have been pursued [40, 42, 43]. Such a strategy may, however, raise a curse of dimensionality for poorly correlated topologies (because the number of reduced (Karhunen-Loève) coordinates serving as the Gaussian germ in the Wiener chaos expansion substantially increases), in which case the propagation must be handled through specific approximation techniques (see the aforementioned references).

3.3.3. Stochastic model for diagonal approximations. In this case, we have $[\boldsymbol{K}(\boldsymbol{x})]=$ $\left[\operatorname{diag}\left(K_{11}(\boldsymbol{x}), K_{22}(\boldsymbol{x})\right)\right]$ for all $\boldsymbol{x}$ in $\Omega$. Let $[\boldsymbol{G}(\boldsymbol{x})]$ be the normalized random matrix such that

$$
[\boldsymbol{K}(\boldsymbol{x})]=\left[\operatorname{diag}\left(\underline{K}_{11}(\boldsymbol{x}) G_{11}(\boldsymbol{x}), \underline{K}_{22}(\boldsymbol{x}) G_{22}(\boldsymbol{x})\right)\right], \quad \forall \boldsymbol{x} \in \Omega .
$$

It follows that

$$
\mathbb{E}\{[\boldsymbol{G}(\boldsymbol{x})]\}=\left[I_{2}\right] .
$$

We then proceed with the construction of the stochastic model for the auxiliary vector-valued field $\left\{\boldsymbol{G}(\boldsymbol{x})=\left(G_{11}(\boldsymbol{x}), G_{22}(\boldsymbol{x})\right), \boldsymbol{x} \in \Omega\right\}$ such that

$$
\mathbb{E}\left\{G_{i i}(\boldsymbol{x})\right\}=1, \quad \forall \boldsymbol{x} \in \Omega, \quad i \in\{1,2\},
$$

and

$$
\sum_{i=1}^{2} \mathbb{E}\left\{\log \left(G_{i i}(\boldsymbol{x})\right)\right\}=s(\boldsymbol{x}),|s(\boldsymbol{x})|<+\infty, \quad \forall \boldsymbol{x} \in \Omega .
$$

By Jensen's inequality, one has

$$
\mathbb{E}\left\{\log \left(G_{i i}(\boldsymbol{x})\right)\right\} \leqslant \log \left(\mathbb{E}\left\{G_{i i}(\boldsymbol{x})\right\}\right)=0
$$

in view of (3.28), for $i \in\{1,2\}$. Consequently, one has

$$
\sum_{i=1}^{2} \mathbb{E}\left\{\log \left(G_{i i}(\boldsymbol{x})\right)\right\} \leqslant 0, \quad \forall x \in \Omega,
$$

and Equation (3.29) can equivalently be recast as

$$
\mathbb{E}\left\{\log \left(G_{i i}(\boldsymbol{x})\right)\right\}=s_{i}(\boldsymbol{x}), \quad\left|s_{i}(\boldsymbol{x})\right|<+\infty, \quad \forall \boldsymbol{x} \in \Omega, \quad i \in\{1,2\} .
$$

Upon introducing these constraints into the maximum entropy principle, it can be deduced that

$$
p_{G_{11}(\boldsymbol{x}), G_{22}(\boldsymbol{x})}\left(g_{1}, g_{2}\right)=\mathbb{I}_{\mathbb{R}_{+}^{*} \times \mathbb{R}_{+}^{*}}\left(g_{1}, g_{2}\right) c(\boldsymbol{x}) \exp \left(-\sum_{i=1}^{2}\left(\lambda_{i}(\boldsymbol{x}) g_{i}+\lambda_{i}^{*}(\boldsymbol{x}) \log \left(g_{i}\right)\right)\right),
$$

where $\mathbb{I}_{\mathcal{S}}$ is the indicator function of the support $\mathcal{S}, c(\boldsymbol{x})$ is the finite normalization constant, $\left\{\lambda_{i}(\boldsymbol{x})\right\}_{i=1}^{2}$ and $\left\{\lambda_{i}^{*}(\boldsymbol{x})\right\}_{i=1}^{2}$ are Lagrange multipliers that can be seen as model parameters. Hence, one has

$$
p_{G_{11}(\boldsymbol{x}), G_{22}(\boldsymbol{x})}\left(g_{1}, g_{2}\right)=\prod_{i=1}^{2} \mathbb{I}_{\mathbb{R}_{+}^{*}}\left(g_{i}\right) c(\boldsymbol{x}) g_{i}^{-\lambda_{i}^{*}(\boldsymbol{x})} \exp \left(-\lambda_{i}(\boldsymbol{x}) g_{i}\right) .
$$


Equation (3.34) shows for a given $\boldsymbol{x}$ fixed in $\Omega, G_{11}(\boldsymbol{x})$ and $G_{22}(\boldsymbol{x})$ are statistically independent and Gamma distributed with respective sets of parameters $\left(k_{11}(\boldsymbol{x}), \theta_{11}(\boldsymbol{x})\right)$ and $\left(k_{22}(\boldsymbol{x}), \theta_{22}(\boldsymbol{x})\right)$, with $k_{i i}(\boldsymbol{x})=1-\lambda_{i}^{*}(\boldsymbol{x})$ and $\theta_{i i}(\boldsymbol{x})=1 / \lambda_{i}(\boldsymbol{x})$. The transformation $\mathcal{T}_{[K]}$ can thus be defined by letting

$$
K_{i i}(\boldsymbol{x})=\underline{K}_{i i}(\boldsymbol{x})\left(F_{\mathcal{G}\left(k_{i i}(\boldsymbol{x}), \theta_{i i}(\boldsymbol{x})\right)}^{-1} \circ \Phi\right)\left(\xi_{i}(\boldsymbol{x})\right), \quad \forall \boldsymbol{x} \in \Omega,
$$

for $i \in\{1,2\}$. Here, it should be recalled that $F_{\mathcal{G}\left(k_{i i}(x), \theta_{i i}(x)\right)}^{-1}$ denotes the inverse cumulative distribution function of the Gamma distribution with hyperparameters $\left(k_{i i}(\boldsymbol{x}), \theta_{i i}(\boldsymbol{x})\right)$ and $\Phi$ is the inverse cumulative distribution function of the normalized Gaussian distribution. Additionally, $\left\{\xi_{i}(x), x \in \Omega\right\}$ is the centered real-valued Gaussian random field, defined by a correlation function $(\boldsymbol{x}, \boldsymbol{y}) \mapsto \mathcal{R}_{i}(\boldsymbol{x}, \boldsymbol{y})$, corresponding to the $i$-th component of the random field $\{\Xi(x), x \in \Omega\}$.

In practice, the earlier stochastic model is used to represent the two macroscopic coefficients $\{[\boldsymbol{A}(\boldsymbol{x})], \boldsymbol{x} \in \Omega\}$ and $\{[\boldsymbol{C}(\boldsymbol{x})], \boldsymbol{x} \in \Omega\}$. In each case, the model is defined by the mean field $\boldsymbol{x} \mapsto[\underline{K}(\boldsymbol{x})]$ and by the fields $\boldsymbol{x} \mapsto\left(k_{i i}(\boldsymbol{x}), \theta_{i i}(\boldsymbol{x})\right)$ and correlation functions $\mathcal{R}_{i}, i \in\{1,2\}$ - which may differ depending on whether $\{[\boldsymbol{A}(\boldsymbol{x})], \boldsymbol{x} \in \Omega\}$ or $\{[\boldsymbol{C}(\boldsymbol{x})], \boldsymbol{x} \in \Omega\}$ is under consideration. In the sequel, these parameters will be obtained by using classical statistical estimators on the numerical database.

3.3.4. Stochastic model for the anisotropic coefficients. We now turn to the case where $\{[\boldsymbol{K}(\boldsymbol{x})], \boldsymbol{x} \in$ $\Omega$ \} is modeled as a $\mathbb{M}_{2}^{+}(\mathbb{R})$-valued random field with anisotropic fluctuations. In this context, applying the previous methodology makes $\left\{[\boldsymbol{K}(\boldsymbol{x})], \boldsymbol{x} \in \Omega\right.$ \} belong to the class $\mathrm{SFE}^{+}$of random fields defined in [37]. For the sake of completeness, we briefly review key aspects and results of this model in the following (we refer the reader to [37] for technical details).

First of all, consider the following matrix decomposition at every single point $x$ of $\Omega$ :

$$
[\boldsymbol{K}(\boldsymbol{x})]=[\underline{L}(\boldsymbol{x})]^{\mathrm{T}}[\boldsymbol{G}(\boldsymbol{x})][\underline{L}(\boldsymbol{x})],
$$

where $[\underline{L}(\boldsymbol{x})]$ corresponds to the upper-triangular matrix involved in the Cholesky decomposition of $[\underline{K}(\boldsymbol{x})]$ (i.e., $\left.[\underline{K}(\boldsymbol{x})]=[\underline{L}(\boldsymbol{x})]^{\mathrm{T}}[\underline{L}(\boldsymbol{x})]\right)$ and $[\boldsymbol{G}(\boldsymbol{x})]$ is a random matrix with values in $\mathbb{M}_{2}^{+}(\mathbb{R})$ such that (3.36)

$$
\mathbb{E}\{[\boldsymbol{G}(\boldsymbol{x})]\}=\left[I_{2}\right], \quad \forall \boldsymbol{x} \in \Omega .
$$

The family $\left\{p_{[\boldsymbol{G}(\boldsymbol{x})]}\right\}_{x \in \Omega}$ of first-order marginal distribution of $\{[\boldsymbol{G}(\boldsymbol{x})], \boldsymbol{x} \in \Omega\}$ is constructed by using Shannon's entropy maximization under the previous mean constraint, as well as under the constraint

$$
\mathbb{E}\{\log (\operatorname{det}([\boldsymbol{G}(\boldsymbol{x})]))\}=s(\boldsymbol{x}),|s(\boldsymbol{x})|<+\infty, \quad \forall \boldsymbol{x} \in \Omega
$$

introduced in Section 3.3.2. It can then be shown that the probability density function of $[\boldsymbol{G}(\boldsymbol{x})]$ (with respect to the measure $\sqrt{2} \mathrm{~d}[G]_{11} \mathrm{~d}[G]_{12} \mathrm{~d}[G]_{22}$, with $d[G]_{i j}$ the Lebesgue measure on $\left.\mathbb{R}\right)$ at point $\boldsymbol{x}$ reads as follows (see [44] for technical details):

$$
\left.p_{[G(\boldsymbol{x})]}([G])\right)=\mathbb{I}_{\mathbb{M}_{2}^{+}(\mathbb{R})}([G]) c(\boldsymbol{x}) \operatorname{det}([G])^{b(\boldsymbol{x})} \exp (-a(\boldsymbol{x}) \operatorname{tr}([G])),
$$

where $c(\boldsymbol{x})$ is the normalization constant, $a(\boldsymbol{x})$ is defined by

$$
a(\boldsymbol{x})=\frac{3}{2 \delta_{[\boldsymbol{G}(\boldsymbol{x})]}^{2}}
$$

and

$$
b(\boldsymbol{x})=\frac{3\left(1-\delta_{[\boldsymbol{G}(\boldsymbol{x})]}^{2}\right)}{2 \delta_{[\boldsymbol{G}(\boldsymbol{x})]}^{2}} .
$$

In the equations given, $\delta_{[\boldsymbol{G}(\boldsymbol{x})]}$ is the coefficient of variation of the random matrix $[\boldsymbol{G}(\boldsymbol{x})]$ at point $\boldsymbol{x}$, defined as in (3.19) and such that

$$
0<\delta_{[G(x)]}<\sqrt{3 / 7} .
$$


This dispersion parameter can be related to the one exhibited by $[\boldsymbol{K}(\boldsymbol{x})]$ through

$$
\delta_{[K(x)]}=\frac{\delta_{[\boldsymbol{G}(x)]}}{\sqrt{3}} \sqrt{1+\frac{\operatorname{tr}([\underline{K}(\boldsymbol{x})])^{2}}{\operatorname{tr}\left([\underline{K}(\boldsymbol{x})]^{2}\right)}} .
$$

Next, it is found convenient - especially for sampling issues - to introduce the factorization

$$
[\boldsymbol{G}(\boldsymbol{x})]=[\boldsymbol{L}(\boldsymbol{x})]^{\mathrm{T}}[\boldsymbol{L}(\boldsymbol{x})],
$$

where $[\boldsymbol{L}(\boldsymbol{x})]$ is a $(2 \times 2)$ upper-triangular real random matrix with statistically independent components. Owing to the change of measure [37, 44], it can be shown that each component can be defined and generated through the following relations:

$$
\begin{gathered}
{[\boldsymbol{L}(\boldsymbol{x})]_{11}=\sqrt{\frac{2}{3}} \delta_{[\boldsymbol{G}(\boldsymbol{x})]} \sqrt{\left(F_{\mathcal{G}\left(k_{11}(\boldsymbol{x}), 1\right)}^{-1} \circ \Phi\right)\left(\xi_{1}(\boldsymbol{x})\right)}, \quad k_{11}(\boldsymbol{x})=\frac{3}{2 \delta_{[\boldsymbol{G}(\boldsymbol{x})]}^{2}},} \\
{[\boldsymbol{L}(\boldsymbol{x})]_{12}=\frac{\delta_{[\boldsymbol{G}(\boldsymbol{x})]}}{\sqrt{3}} \xi_{2}(\boldsymbol{x}),} \\
{[\boldsymbol{L}(\boldsymbol{x})]_{22}=\sqrt{\frac{2}{3}} \delta_{[\boldsymbol{G}(\boldsymbol{x})]} \sqrt{\left(F_{\mathcal{G}\left(k_{22}(x), 1\right)}^{-1} \circ \Phi\right)\left(\xi_{3}(\boldsymbol{x})\right)}, \quad k_{22}(\boldsymbol{x})=\frac{3}{2 \delta_{[\boldsymbol{G}(\boldsymbol{x})]}^{2}}+\frac{1}{2},}
\end{gathered}
$$

where $\left\{\xi_{i}(x), x \in \Omega\right\}, 1 \leqslant i \leqslant 3$, are the independent components of the centered Gaussian random field $\{\Xi(x), x \in \Omega\}$ with values in $\mathbb{R}^{3}$ and defined by the matrix-valued (normalized) covariance function:

$$
[\mathcal{R}(\boldsymbol{x}, \boldsymbol{y})]=\mathbb{E}\{\Xi(\boldsymbol{x}) \otimes \Xi(\boldsymbol{y})\}=\left[\operatorname{diag}\left(\mathcal{R}_{1}(\boldsymbol{x}, \boldsymbol{y}), \mathcal{R}_{2}(\boldsymbol{x}, \boldsymbol{y}), \mathcal{R}_{3}(\boldsymbol{x}, \boldsymbol{y})\right)\right], \quad \forall(\boldsymbol{x}, \boldsymbol{y}) \in \Omega \times \Omega,
$$

with $[\mathcal{R}(\boldsymbol{x}, \boldsymbol{x})]=\left[I_{3}\right], \forall \boldsymbol{x} \in \Omega$. Substituting (3.45-3.47) and (3.44) into (3.36) allows us to define the mapping $\mathcal{T}_{[\boldsymbol{K}]}$ and provides an efficient sampling algorithm for the random field $\{[\boldsymbol{K}(\boldsymbol{x})], \boldsymbol{x} \in \Omega\}$.

This stochastic model is then defined by the mean field $\boldsymbol{x} \mapsto[K(\boldsymbol{x})]$, by the dispersion field $\boldsymbol{x} \mapsto \delta_{[\boldsymbol{G}(\boldsymbol{x})]}$ and by the correlation functions $\mathcal{R}_{i}, i \in\{1,2,3\}$. As in Section 3.3.3, these parameters are computed from the database by using classical statistical estimators (the convergence of which was controlled).

3.3.5. Comparing the predictions of the peak pressure and load capacity. Selecting the peak pressure $p_{0}^{\max }$ as the first macroscopic QoI, we compare in the following the predictions delivered by the two stochastic models with the reference results. As previously mentioned, the hyperparameters of each model were estimated from the database, and the convergence of each estimator (with respect to the number of realizations) was checked. The probability density function of $p_{0}^{\max }$ is shown for each case in Figure 16 for $R=10, \eta=10$, and $\delta=0.45$.

It is seen that the two models deliver fairly good predictions for the probability density function of $p_{0}^{\max }$. More specifically, the predictions of the mean peak pressure are equal to $22.2427 \mathrm{MPa}$ and 22.3570 MPa for the anisotropic and diagonal models, respectively, with a reference value equal to $22.8825 \mathrm{MPa}$. The relative errors in the mean are therefore equal to $2.8 \%$ and $2.3 \%$ for the aforementioned models, respectively.

By considering the load capacity as the second QoI, we compare in Figure 17 the predictions of the mean pressure fields.

Here again, we observe that the probabilistic models allow the mean pressure fields and hence the mean load capacity to be well predicted. Because the estimated mean fields are almost indistinguishable, the plots of the relative error between the reference field and the one predicted by resorting on any of the two stochastic models are further depicted in Figure 18.

In accordance with Figure 17, the relative error turns out to be small regardless of the probabilistic representations of the macroscopic coefficients. Moreover, it is seen that the diagonal random fields 


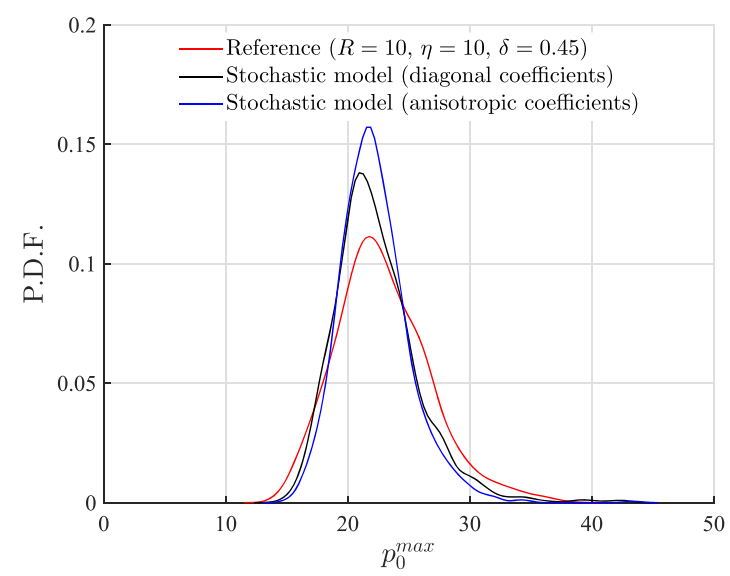

Figure 16. A comparison of the prediction of the probability density function for the peak pressure $p_{0}^{\max }$ is proposed. The reference solution is shown in black, while the solutions obtained with the stochastic models are depicted in black (diagonal model) and blue (anisotropic model).

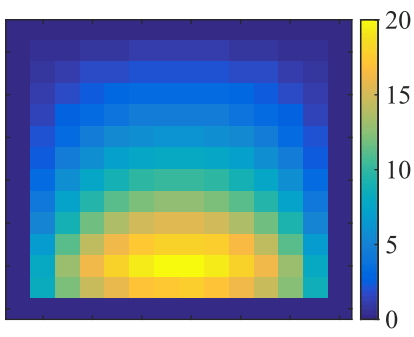

(a) Reference solution

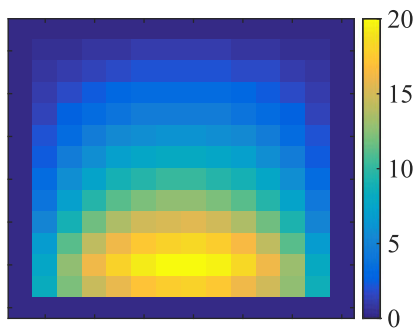

(b) Stochastic model introduced in Section 3.3.3

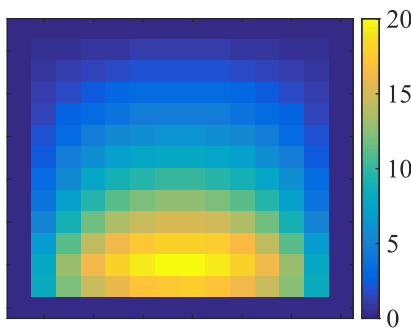

(c) Stochastic model introduced in Section 3.3.4

Figure 17. In this figure, the predictions of the mean pressure field (which is related to the mean load capacity) are compared.

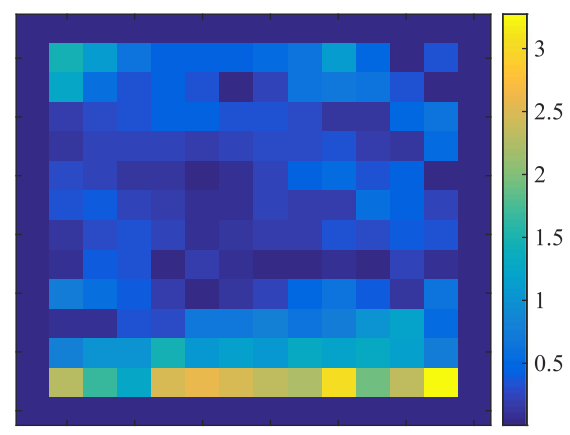

(a) Error relative (\%) using the diagonal model

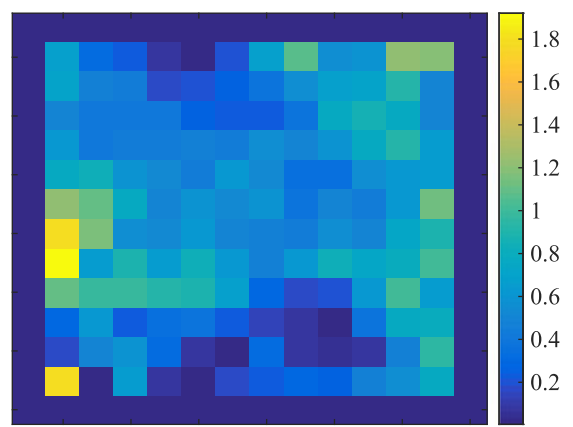

(b) Error relative (\%) using the anisotropic model

Figure 18. Here, the relative errors between the reference and predicted mean pressure fields are shown.

generate a larger discrepancy at the locations where the fields exhibit large statistical fluctuations. This demonstrates the relevance of properly modeling the anisotropic fluctuations for the case under study. These results show that for the random topography under consideration, accurately reproducing the second-order statistics of the homogenized coefficients $\{[\boldsymbol{A}(\boldsymbol{x})], \boldsymbol{x} \in \Omega\}$ and $\{[\boldsymbol{C}(\boldsymbol{x})], \boldsymbol{x} \in \Omega\}$ is, indeed, sufficient to predict the previous key macroscopic quantities. 


\section{CONCLUSION}

The ability to efficiently and effectively capture the influence of the microstructure on the macroscopic response of structures and interfaces is a central problem in multiscale mechanics. In view of the typically very small length scales associated with the microstucture, two-scale formulations based on averaging and homogenization approaches have been the driving force towards the construction of suitable numerical analysis frameworks. However, when the microstucture is random, the predictive capability of these approaches may diminish in comparison with the setting where ideal periodicity applies. The goal of the present study was to study and address the influence of randomness in the context of a particular class of multiscale interface problems, namely, hydrodynamic lubrication with surface roughness. Representative numerical studies have demonstrated the significant influence of the specific roughness realization on the interface response even at very small ratios of a representative microscopic length scale to a macroscopic one, thereby highlighting the stochastic nature of the problem. In order to account for this influence effectively, a stochastic multiscale analysis framework was developed where local upscaling relies on homogenization theory. The influence of major coarse-scale and fine-scale analysis parameters on the predictive capability of this framework was demonstrated through local and global quantities of interest such as the peak pressure and the load capacity, respectively. Coarse-scale stochastic models of the interface response were additionally constructed, with an ability to consider local anisotropy features, which are important from a practical point of view where it is of interest to rapidly and accurately estimate mean values and standard deviations in quantities of interest. Overall, the presented stochastic multiscale framework offers a high predictive capability for global quantities of interest and a controllable error margin on local quantities of interest.

Although the present study addressed the stochastic nature of hydrodynamic lubrication problems due to randomness in the microscopic surface topology, the periodic setting can also benefit from a similar approach. A specific case of interest is micro-texture design [20] where one would like to account for uncertainties in the multiscale design objectives or in the macroscopic operating conditions of the interface. Alternatively, periodic or random imperfections and inconsistencies because of manufacturing operations lead to variations in the statistical characteristics of a surface, for instance in its root-mean-square roughness, and therefore to subsequent variability in the macroscopic performance. Such variations are amenable to a multiscale stochastic analysis based on the presented framework. Finally, in view of the significant role that surface roughness or texturing plays in closely related interface problems such as friction physics [45-47], it would be desirable to develop a class of approaches, which could address stochastic aspects in multiscale tribology problems in a broader context. Such studies would contribute to a better understanding of the influence of microscopic heterogeneities on the physically observed interface response and additionally help bridge the gap between theoretical predictions and experimental variations.

\section{ACKNOWLEDGEMENTS}

İ. Temizer acknowledges support by the Scientific and Technological Research Council of Turkey (TÜBİTAK) under the 1001 Program (Grant no. 114M406).

\section{REFERENCES}

1. Hamrock B, Schmid S, Jacobson B. Fundamentals of Fluid Film Lubrication. CRC Press: Boca Raton, 2004.

2. Szeri AZ. Fluid Film Lubrication. Cambridge University Press: New-York, 2011.

3. Bayada G, Ciuperca I, Jai M. Homogenized elliptic equations and variational inequalities with oscillating parameters. Application to the study of thin flow behavior with rough surfaces. Nonlinear Analysis: Real World Applications 2006; 7(5):950-966.

4. Prat M, Plouraboué F, Letalleur N. Averaged Reynolds equation for flows between rough surfaces in sliding motion. Transport in Porous Media 2002; 48:291-313.

5. Greene S, Liu Y, Chen W, Liu WK. Computational uncertainty analysis in multiresolution materials via stochastic constitutive theory. Computer Methods in Applied Mechanics and Engineering 2011; 200:309-325.

6. Ostoja-Starzewski M. Random field models of heterogeneous materials. International Journal of Solids and Structures 1998; 35:2429-2455. 
7. Patir N, Cheng HS. An average flow model for determining effects of three-dimensional roughness on partial hydrodynamic lubrication. Journal of Lubrication Technology, Transactions of the ASME 1978; 100:12-17.

8. Patir N, Cheng HS. Application of average flow model to lubrication between rough sliding surfaces. Journal of Lubrication Technology, Transactions of the ASME 1979; 101:220-230.

9. Elrod HG. A general theory for laminar lubrication with reynolds roughness. Journal of Lubrication Technology 1979; 101:8-14.

10. Tripp JH. Surface roughness effects in hydrodynamic lubrication: the flow factor method. Journal of Lubrication Technology, Transactions of the ASME 1983; 105:458-463.

11. Kabacaoğlu G, Temizer İ. Homogenization of soft interfaces in time-dependent hydrodynamic lubrication. Computational Mechanics 2015; 56:421-441.

12. Nemat-Nasser S, Hori M. Micromechanics: Overall Properties of Heterogeneous Materials. North-Holland: Amsterdam, 1991.

13. Torquato S. Random Heterogeneous Materials: Microstructure and Macroscopic Properties. Springer: Berlin Heidelberg New York, 2002.

14. Almqvist A, Lukkassen D, Meidell A, Wall P. New concepts of homogenization applied in rough surface hydrodynamic lubrication. International Journal of Engineering Science 2007; 45:139-154.

15. Sahlin F, Larsson R, Almqvist A, Lugt PM, Marklund P. A mixed lubrication model incorporating measured surface topography. Part 1: theory of flow factors. Proceedings of the Institution of Mechanical Engineers, Part J: Journal of Engineering Tribology 2009; 24:335-351.

16. Sanchez-Palencia E. Non-homogeneous Media and Vibration Theory. Springer-Verlag: Berlin Heidelberg, 1980.

17. Tartar L. The General Theory of Homogenization: A Personalized Introduction. Springer-Verlag: Berlin Heidelberg, 2009.

18. Braides A, Defranceschi A. Homogenization of Multiple Integrals. Oxford: New-York, 1999.

19. Efendiev Y, Hou TY. Multiscale Finite Element Methods. Springer-Verlag: New-York, 2009.

20. Waseem A, Temizer İ, Kato J, Terada K. Homogenization-based design of surface textures in hydrodynamic lubrication. International Journal for Numerical Methods in Engineering 2016; 108:1427-1450.

21. Bayada G, Chambat M. Homogenization of the Stokes system in a thin film flow with rapidly varying thickness. Mathematical Modeling and Numerical Analysis 1989; 23:205-234.

22. Pérez-Ràfols F, Larsson R, Lundström S, Wall P, Almqvist A. A stochastic two-scale model for pressure-driven flow between rough surfaces. Proceedings of the Royal Society A 2016; 472:20160069(1-20).

23. Guilleminot J, Soize C, Ghanem RG. Stochastic representation for anisotropic permeability tensor random fields. International Journal for Numerical and Analytical Methods in Geomechanics 2012; 36:1592-1608.

24. Stout KJ, Sullivan PJ, Dong WP, Manisah E, Luo N, Mathia T, Zahouani H. The development of methods for the characterization of roughness in three dimensions. Technical report, Commission of the European Communities, 1993.

25. Buczkowski R, Kleiber M. Statistical models of rough surfaces for finite element 3D-contact analysis. Archives of Computational Methods in Engineering 2009; 16:399-424.

26. Jiang X, Scott PJ, Whitehouse DJ, Blunt L. Paradigm shifts in surface metrology. Part I. Historical philosophy. Proceedings of the Royal Society of London A: Mathematical, Physical and Engineering Sciences 2007; 463(2085):2049-2070.

27. Jiang X, Scott PJ, Whitehouse DJ, Blunt L. Paradigm shifts in surface metrology. Part II. The current shift. Proceedings of the Royal Society of London A: Mathematical, Physical and Engineering Sciences 2007; 463(2085):2071-2099.

28. Whitehouse DJ. Fractal or fiction. Wear 2001; 249:345-353.

29. Zahouani H, Vargiolu R, Loubet J-L. Recent advances in contact mechanics fractal models of surface topography and contact mechanics. Mathematical and Computer Modelling 1998; 28:517-534.

30. Adler RJ, Firman D. A non-Gaussian model for random surfaces. Philosophical Transactions of the Royal Society of London. Series A, Mathematical and Physical Sciences 1981; 303:433-462.

31. McCool JI. Non-Gaussian effects in microcontact. International Journal of Machine Tools and Manufacture 1992; 32:115-123.

32. Adler RJ, Taylor JE. Random Fields and Geometry, Springer Monographs in Mathematics. Springer: New York, 2007.

33. Rasmussen CE, Williams CKI. Gaussian Processes for Machine Learning (Adaptive Computation and Machine Learning). The MIT Press: Cambridge, 2005.

34. Jaynes ET. Information theory and statistical mechanics. Physical Review 1957; 106/108(4/2):620-630/171-190.

35. Shannon CE. A mathematical theory of communication. Bell System Technical Journal 1948; 27:379-423/623-659.

36. Guilleminot J, Soize C. Stochastic model and generator for random fields with symmetry properties: application to the mesoscopic modeling of elastic random media. Multiscale Modeling \& Simulation 2013; 11(3):840-870.

37. Soize C. Non-Gaussian positive-definite matrix-valued random fields for elliptic stochastic partial differential operators. Computer Methods in Applied Mechanics and Engineering 2006; 195:26-64.

38. Guilleminot J, Soize C. Itô SDE-based generator for a class of non-Gaussian vector-valued random fields in uncertainty quantification. SIAM Journal on Scientific Computing 2014; 36(6):A2763-A2786.

39. Nouy AA, Soize C. Random fields representations for stochastic elliptic boundary value problems and statistical inverse problems. European Journal of Applied Mathematics 2014; 25(3):339-373.

40. Arnst M, Ponthot J-P. An overview of nonintrusive characterization, propagation, and sensitivity analysis of uncertainties in computational mechanics. International Journal for Uncertainty Quantification 2014; 4(5):387-421.

41. Kamiński M. The Stochastic Perturbation Method for Computational Mechanics. John Wiley \& Sons: Chichester, 2013. 
42. Le Maître OP, Knio OM. Spectral Methods for Uncertainty Quantification with Applications to Computational Fluid Dynamics. Springer: Dordrecht, 2010.

43. Sullivan T. Introduction to Uncertainty Quantification. Springer: Cham, 2015.

44. Soize C. A nonparametric model of random uncertainties for reduced matrix models in structural dynamics. Probabilistic Engineering Mechanics 2000; 15:277-294.

45. Kılıç Kİ, Temizer İ. Tuning macroscopic sliding friction at soft contact interfaces: interaction of bulk and surface heterogeneities. Tribology International 2016; 104:83-97.

46. Murarash B, Itovich Y, Varenberg M. Tuning elastomer friction by hexagonal surface patterning. Soft Matter 2011; 7:5553-5557.

47. Persson BNJ. Sliding Friction (2nd edn). Springer-Verlag: Berlin Heidelberg New York, 2000. 